\title{
Metabolomics in Alzheimer's disease: The need of complementary analytical platforms for the identification of biomarkers to unravel the underlying pathology
}

\author{
Raúl González-Domínguez $^{\mathrm{a}, \mathrm{b}, *}$, Ana Sayago ${ }^{\mathrm{a}, \mathrm{b}}$, Ángeles Fernández-Recamales ${ }^{\mathrm{a}, \mathrm{b}}$ \\ a Department of Chemistry, Faculty of Experimental Sciences, University of Huelva, 21007, Spain \\ ${ }^{\mathrm{b}}$ International Campus of Excellence CeiA3, University of Huelva, 21007, Spain
}

\section{A R T I C L E IN F O}

\section{Article history:}

Received 29 October 2016

Received in revised form 27 January

2017

Accepted 5 February 2017

Available online $\mathrm{xxx}$

Keywords:

Alzheimer's disease

Metabolomics

Complementary platforms

Mass spectrometry

Nuclear magnetic resonance

\section{A B S T R A C T}

Alzheimer's disease is a complex neurodegenerative disorder characterized by a multi-factorial etiology, not completely understood to date. In this context, the application of metabolomics is emerging in the last years because of its potential to monitor molecular alterations associated with disease pathogenesis and progression, as well as to discover candidate diagnostic biomarkers. However, the huge heterogeneity and dynamism of the human metabolome makes impossible the simultaneous determination of the entire set of metabolites from complex biological samples. Thus, the most common strategy to get a comprehensive overview of the organism's phenotypic expression is the combined use of complementary metabolomic platforms. In this review, we summarize the advantages and limitations of the most important analytical techniques usually employed in metabolomics, including nuclear magnetic resonance, direct infusion mass spectrometry and hyphenated approaches based on the coupling of orthogonal separation mechanisms (i.e. liquid chromatography, gas chromatography, capillary electrophoresis) with mass spectrometry. Moreover, the suitability of metabolomics to unravel the complex pathology underlying to Alzheimer's disease is also presented.

(C) 2016 Published by Elsevier Ltd.

Abbreviations: A $\beta$, amyloid-beta; AD, Alzheimer's disease; ALS, amyotrophic lateral sclerosis; APCI, atmospheric pressure chemical ionization; APOE, apolipoprotein E; APP, amyloid precursor protein; APPI, atmospheric pressure photoionization; CE, capillary electrophoresis; CSF, cerebrospinal fluid; D-Gal, D-galactose; DIMS, direct infusion mass spectrometry; ECA, electrochemical array; EI, electron impact; ESI, electrospray ionization; FI, flow injection; FTLD, frontotemporal lobe dementia; GC, gas chromatography; HA, huperzine A; HC, healthy control; HILIC, hydrophilic interaction liquid chromatography; HMDB, human metabolome database; HPLC, high-performance liquid chromatography; KXS, Kai-Xin-San; LBD, Lewy body disease; LC, liquid chromatography; LMW, low molecular weight; LP, ligustrazine phosphate; LPL, lyso-phospholipid; MAGL, monoacylglycerol lipase; MCI, mild cognitive impairment; MDMS-SL, multi-dimensional mass spectrometry-based shotgun lipidomics; MRS, magnetic resonance spectroscopy; MS, mass spectrometry; NMR, nuclear magnetic resonance; PC, phosphatidylcholine; PD, Parkinson's disease; PL, phospholipid; PS1, presenilin 1; PUFA, polyunsaturated fatty acid; ROC, receiver operating characteristic; RP, reversed phase; SAMP8, senescence accelerated mouse-prone 8; SAMR1, senescence accelerated mouse-resistant 1; SCI, subjective cognitive impairment; Sco, scopolamine; SFA, saturated fatty acid; TCA, tricarboxylic acid cycle; TG, total gingenosides; UHPLC, ultra-high-performance liquid chromatography; WT, wild type

* Corresponding author. Present address: Biomarkers \& Nutrimetabolomic Lab, Department of Nutrition, Food Sciences and Gastronomy, Food Technology Reference Net (XaRTA), Nutrition and Food Safety Research Institute (INSA), Faculty of Pharmacy and Food Sciences, University of Barcelona. 08028, Spain.

Email addresses: raul.gonzalez@dqcm.uhu.es (R. González-Domínguez); ana. sayago@dqcm.uhu.es (A. Sayago); recamale@dqcm.uhu.es (Á.

Fernández-Recamales) 


\section{Introduction to Alzheimer's disease}

In the last decades, Alzheimer's disease (AD) has become a major challenge for the public health system due to the dramatically increase of aged population worldwide. This neurodegenerative disorder, clinically defined by an insidious onset and a progressive loss of memory and other cognitive functions, is the most prevalent form of dementia among elderly people, accounting for $70 \%$ of total cases [1]. This cognitive and functional decline is preceded by a pre-clinical phase known as mild cognitive impairment (MCI), a complex syndrome characterized by memory failures that may be considered as an intermediate stage in the development of $\mathrm{AD}$ from normal aging [2]. The exact etiology of AD is still a huge puzzle to be solved in which multiple players participate, including genetic, environmental, as well as ageing-related factors. In this sense, there is international consensus about the multifactorial nature of this disorder, involving numerous biochemical perturbations such as the deposition of $\beta$-amyloid plaques $(A \beta)$, hyperphosphorylation of tau protein, oxidative stress, inflammation, abnormal metal homeostasis, as well as disruption in energetic and neurotransmitter pathways, among others [3-6]. Furthermore it should be noted that, although AD has traditionally been considered a primary neurodegenerative disorder that specifically affects the central nervous system, there is growing evidence that pathological processes associated with $\mathrm{AD}$ can also manifest in the peripheral system [7].

Nowadays, diagnosis of AD can only be performed via exclusion of other pathologies by using a combination of different neuropsychological and laboratory tests [8]. However, definitive diagnosis requires the post-mortem examination of the brain in order to confirm the presence of $A \beta$ plaques and neurofibrillary tangles. Furthermore, this diagnostic protocol is only effective in patients with significant cognitive decline, which impossibilities an early detection of $\mathrm{AD}$ and reduces the efficacy of subsequent pharmacological treatments. Finally, it has been demonstrated that these criteria show low specificity against other dementia (70\%), with moderate sensitivity (80\%) [9]. For all these reasons, the identification of new AD biomarkers is critical in order to monitor molecular mechanisms associated with disease pathogenesis and progression, discover early and accurate diagnostic tests, as well as for helping in the development of novel drug treatments. To this end, the application of systems biology technologies, and particularly metabolomics, is emerging in recent years because of their potential to investigate biochemical alterations underlying to pathological processes in a comprehensive manner.

\section{The potential of metabolomics for the identification of disease biomarkers}

Metabolomics relies on the non-targeted characterization of the entire set of metabolites from a biological sample. A major strength of this approach is its potential to provide a global overview of multiple biochemical pathways, reflecting changes downstream of the genomic, transcriptomic and proteomic levels [10]. In this context, it is noteworthy that metabolites play a prominent role in systems biology and the final phenotypic expression of the organism, which confers to metabolomics a number of advantages over other traditional omic-techniques such as genomics and proteomics, as summarized below.

- Metabolites are the most reliable indicators of the organism's phenotype, given that represent the end point of biochemical reactions, directly reflecting the interactions between gene expression, proteins function and the environment [10].

- Changes in metabolite concentrations are generally more pronounced than those observed in other biological levels such as the transcriptome or the proteome, making the detection of metabolite profiles a relatively sensitive measure of biological status [11].

- Metabolic alterations are rapid indicators of abnormalities occurring in the organism due to the high dynamism of metabolite homeostasis [12].

- Metabolomic data can be directly translated across species because most metabolic pathways are highly conserved through evolution, which is crucial in order to inter-relate findings from experiments with laboratory animals and human studies [13].

- Metabolomics is applicable to a variety of easily accessible biological samples such as blood, urine or cerebrospinal fluid (CSF), with reduced cost and analysis time, thus highlighting the clinical utility of this approach [14].

Thus, metabolomics has been proven to be a suitable tool in biomedical research for the investigation of complex phenotypes affected by genetic and environmental factors. Particularly, this holistic approach plays a pivotal role in the study of multifactorial disorders such as Alzheimer's disease, in which many heterogeneous processes are involved. However, the great complexity of the human metabolome considerably hinders the identification of these metabolic features in a comprehensive manner. The human metabolome has not been fully characterized yet, but recent estimates are over 40,000 annotated compounds, according to the Human Metabolome Database (HMDB; http://www.hmdb.ca) [15]. In this sense, it should be noted that the metabolome comprises compounds from various origins. Endogenous metabolites are synthetized and metabolized within the organism by means of the action of enzymes involved in catabolic and anabolic reactions. On the other hand, gut microflora also generates a great number of compounds which can exhibit a large impact on health and disease status. Finally, exogenous metabolites or xenobiotics are imported into the organism from different sources (e.g. drugs, food, pollutants), and then are usually bio-transformed in order to facilitate their excretion, thus generating a myriad of compounds detectable in different biofluids and tissues. Another pitfall in metabolomics is the great compositional diversity of the metabolome. Unlike genes, transcripts and proteins, which are biopolymers that share the same building blocks (four nucleotides for DNA/RNA; twenty amino acids in the case of proteins), metabolites can be categorized into several very different chemical classes, including lipids, carbohydrates, amino acids, peptides, organic acids, and other many low molecular weight (LMW) compounds, which results in a high variability of physicochemical properties [12]. Thereby, metabolic profiles are composed of metabolites in a wide range of molecular weights, from $1 \mathrm{Da}$ (proton) to $1500-2000 \mathrm{Da}$ (complex lipids and small peptides); polarities, including hydrophilic LMW-metabolites as well as hydrophobic lipids; and volatilities, from low boiling point metabolites (e.g. breath metabolites) to heavier compounds. Furthermore, metabolites are present in biological samples in wide range of concentrations, which span over several orders of magnitude (from molar to sub-picomolar), and are subjected to a high temporal, spatial and inter-individual variability depending on age, gender or diet, among other factors [12]. Consequently, a great challenge of current metabolomic research is the development of novel analytical approaches with the aim to maximize metabolite coverage.

In recent years, numerous efforts have been made in order to increase the capabilities of metabolomics for the identification of as many metabolites as possible, with an especial focus on two complementary research lines: (i) development of algorithms and databases for the annotation of metabolites; and (ii) optimization of orthogonal analytical platforms to expand metabolomic coverage. The annotation of discriminant metabolites is usually based on the querying of exper- 
imental data against metabolomic databases, but most of them are still under development. Thus, the identification of "unknowns" is still widely recognized as a major bottleneck in metabolomics. However, this subject is out of the scope of the present work, so interested readers can resort to a number of excellent recently published reviews [16-18]. On the other hand, considering the great complexity of the metabolome, it is clear that none analytical platform is able to determine the totality of metabolites present in a sample in a single analysis. For this reason, the application of multiple complementary approaches has become the most common strategy with the aim to overcome the analytical bias introduced by these individual techniques.

\section{The application of metabolomics in Alzheimer's disease research}

Alzheimer's disease research has traditionally been hindered because of the long pre-symptomatic period of this neurodegenerative disorder, the impossibility of studying microscopic changes until final stages, as well as the complexity of etiology and the variability of clinical symptoms. Furthermore, the lack of accurateness of current diagnostic tests, especially at early stages of disease, may result in the inclusion of pre-clinical AD patients within control groups in clinical trials, thus limiting the reliability of discovered biomarkers [19]. Thereby, the use of metabolomics has emerged in recent years for the investigation of pathological mechanisms underlying to this disorder and the identification of novel biomarkers. In this context, numerous authors have proposed the application of multiple metabolomic techniques for studying $\mathrm{AD}$ pathogenesis, in both human cohorts and different mouse models (Table 1). With regards to these animal models, it should be noted that the most commonly employed are based on the over-expression of mutated forms of the human amyloid precursor protein (APP), including the $\mathrm{APP}_{\mathrm{Tg} 2576}, \mathrm{APP}_{\mathrm{V} 717 \mathrm{~F}}$ and CRND8 transgenic lines. On the other hand, some mouse models also co-express the mutated human presenilin 1 (PS1) in order to accelerate amyloid deposition, such as the APP $\times$ PS1 or the TASTPM double transgenic models. Moreover, various biological samples have been previously investigated with the aim to get a global overview of pathological changes underlying to AD. Brain tissue analysis shows a great potential since allows the in situ characterization of neuropathological processes associated with AD. Alternatively, cerebrospinal fluid (CSF) is an interesting biofluid given that its composition directly reflects the brain metabolic production. Finally, the use on non-invasive samples, such as blood serum or plasma, urine and saliva, has also been described with the aim to identify simpler and cheaper biomarkers translatable to the clinical practice. A comprehensive summary of previous metabolomic studies in $\mathrm{AD}$ research will be provided in the next sections of this review article, with a special focus on the limitations and complementarities of employed analytical platforms.

\subsection{High-throughput metabolomic approaches}

\subsubsection{Nuclear magnetic resonance}

Nuclear magnetic resonance has played a prominent role in the early beginnings of metabolomics because of its excellent robustness, reproducibility and high-throughput capabilities. In this context, the application of in vivo neuroimaging techniques based on magnetic resonance spectroscopy (MRS) could be considered as the first metabolomic approximation to AD. This tool has successfully been employed for the investigation of $\mathrm{AD}$ pathogenesis and its progression form MCI [20,21], as well as for the characterization of age related changes in various mouse models of this neurodegenerative dis- order [22,23]. Common metabolic features identified by in vivo MRS include reduced brain levels of glutamate and glutamine, increased myo-inositol content, which may be indicative of osmotic stress and astrogliosis, as well as down-regulated $N$-acetyl-aspartate production, a conventional marker of neural integrity. However, the low sensitivity of this analytical approach considerably limits the number of detectable metabolites, thus hindering its implementation in global metabolomic studies.

On the other hand, in vitro NMR can be directly applied onto tissue extracts and biofluids with improved sensitivity, thus enabling the simultaneous identification of up to 100 metabolites. Similarly to the previously described MRS-based investigations, numerous authors proposed the application of this analytical platform to characterize metabolic perturbations associated with $\mathrm{AD}$ in post-mortem brain samples. In the 1990's, several early studies were performed in order to quantify the content of some important neurochemicals in different brain regions and thus confirm previous findings obtained by in vivo MRS [24-26]. More recently, a non-targeted NMR-based metabolomic approach was employed to compare cortical metabolite profiles from $\mathrm{AD}$ and amyotrophic lateral sclerosis (ALS) patients [27]. In this line, Graham et al. also found significant metabolomic alterations in the neocortex region from demented patients, and demonstrated that paired metabolites ratios could be more powerful tools for discriminating AD patients from healthy control (HC) subjects [28]. However, it should be noted that the use of post-mortem tissues implies that disease is at its ending phase, which makes impossible the investigation of disease pathogenesis and progression from early stages. Alternatively, various transgenic animal models are also available for studying the neuropathology associated with AD, from which brain samples can be obtained at different stages of disease. Dedeoglu et al. compared in vivo MRS and in vitro NMR to investigate the differences in the neurochemical profile between $\mathrm{APP}_{\mathrm{Tg} 2576}$ transgenic mice and wild type (WT) littermates, thus demonstrating that a wider range of compounds can be measured by using high resolution spectroscopy (Fig. 1) [29]. Metabolomic analysis of cerebellum samples from this animal model also showed significant alterations in levels of important neurochemicals, such as N-acetyl-aspartate, $\gamma$-aminobutyric acid or glutamate, among others [30]. On the other hand, Forster et al. examined longitudinal metabolic changes in whole brain extracts from TASTPM transgenic mice aged between 3 and 18 months, and surprisingly did not find significant differences in $\mathrm{N}$-acetyl-aspartate levels [31]. Complementarily, other authors proposed the comparative investigation of different brain areas in order to elucidate the regional specificity of characteristic neuropathological mechanisms associated with AD [32-34]. Thus, it has been demonstrated that hippocampus and cortex are the most affected areas by $\mathrm{AD}$-induced perturbations, but significant metabolic impairments can also be observed in other brain regions such as cerebellum, striatum and midbrain.

The analysis of biofluids is an interesting alternative to the use of brain samples with the aim to discover potential diagnostic biomarkers for their implementation in the clinical practice. In this context, the application of NMR-based metabolomics to CSF samples has demonstrated a great utility to discriminate between AD patients and healthy controls because of its close similarity with the brain composition [35-37]. Alternatively, the use of peripheral samples also shows a great potential for the development of non-invasive diagnostic tests. In this sense, Graham et al. employed ${ }^{1} \mathrm{H}$ NMR to compare metabolomic alterations in brain and plasma samples from the APP $\times$ PS1 mouse model [38]. Despite metabolites in plasma were present in lower concentrations and were subjected to a higher variability, they observed that plasma metabolite profiles provide more 
Table 1

Summary of metabolomic studies in Alzheimer's disease research.

\begin{tabular}{|c|c|c|c|}
\hline study population & sample & findings & Ref. \\
\hline \multicolumn{4}{|l|}{ Nuclear Magnetic Resonance } \\
\hline $\mathrm{AD}(\mathrm{N}=12), \mathrm{HC}(\mathrm{N}=5)$ & whole brain & $\downarrow N$-acetyl-aspartate, $\gamma$-aminobutyric acid; $\uparrow$ glutamate & [24] \\
\hline $\mathrm{AD}(\mathrm{N}=13), \mathrm{HC}(\mathrm{N}=4)$ & temporoparietal cortex & $\downarrow N$-acetyl-aspartate, creatine, $\gamma$-aminobutyric acid & [25] \\
\hline $\mathrm{AD}(\mathrm{N}=13), \mathrm{HC}(\mathrm{N}=4)$ & hippocampus, cerebellum & $\downarrow N$-acetyl-aspartate, $\gamma$-aminobutyric acid & [26] \\
\hline $\operatorname{AD}(\mathrm{N}=8), \operatorname{ALS}(\mathrm{N}=11)$ & frontal cortex & $\uparrow$ alanine, acetate, glutamate, glutamine; $\downarrow$ lactate, creatine & [27] \\
\hline $\mathrm{AD}(\mathrm{N}=15), \mathrm{HC}(\mathrm{N}=15)$ & neocortex & $\uparrow$ alanine, taurine & [28] \\
\hline $\mathrm{APP}_{\mathrm{Tg} 2576}(\mathrm{~N}=9), \mathrm{WT}(\mathrm{N}=10)$ & frontal cortex & $\downarrow N$-acetyl-aspartate, glutamate, glutathione; $\uparrow$ taurine & [29] \\
\hline $\mathrm{APP}_{\mathrm{Tg} 2576}(\mathrm{~N}=9), \mathrm{WT}(\mathrm{N}=8)$ & cerebellum & $\uparrow$ taurine, creatine, glutamate, $N$-acetyl-aspartate, $\gamma$-aminobutyric acid & {$[30]$} \\
\hline $\begin{array}{l}\text { TASTPM }(\mathrm{N}=7-8), \text { WT } \\
(\mathrm{N}=5-9)\end{array}$ & whole brain & $\uparrow$ myo-inositol, choline, glycerophosphocholine; $\downarrow$ succinate & [31] \\
\hline $\begin{array}{l}\text { CRND8 }(\mathrm{N}=5 / 5 \text { young/old }) \\
\text { WT }(\mathrm{N}=4 / 8 \text { young } / \text { old })\end{array}$ & $\begin{array}{l}\text { cortex, frontal cortex, } \\
\text { cerebellum, } \\
\text { hippocampus, striatum, } \\
\text { pons, midbrain, olfactory } \\
\text { bulb }\end{array}$ & $\begin{array}{l}\downarrow N \text {-acetyl-aspartate, glutamate, glutamine, taurine, } \gamma \text {-aminobutyric acid, choline, phosphocholine, creatine, } \\
\text { phosphocreatine, succinate; } \uparrow \text { lactate, aspartate, glycine, alanine, leucine, isoleucine, valine, free fatty } \\
\text { acids }\end{array}$ & [32] \\
\hline $\begin{array}{l}\text { APP } \times \text { PS1 }(N=10), \text { WT } \\
(N=10)\end{array}$ & $\begin{array}{l}\text { cortex (frontal, occipital, } \\
\text { parietal, temporal), } \\
\text { thalamus, hippocampus, }\end{array}$ & $\downarrow N$-acetyl-aspartate; $\uparrow$ myo-inositol, scyllo-inositol & [33] \\
\hline $\mathrm{APP}_{\mathrm{Tg} 2576}(\mathrm{~N}=39), \mathrm{WT}(\mathrm{N}=44)$ & $\begin{array}{l}\text { frontal cortex, rhinal } \\
\text { cortex, hippocampus, } \\
\text { cerebellum, midbrain }\end{array}$ & $\downarrow$ glutamate, $N$-acetyl-aspartate, $m y o$-inositol, phosphocholine, $\gamma$-aminobutyric acid; $\uparrow$ creatine, taurine & [34] \\
\hline $\mathrm{AD}(\mathrm{N}=20), \mathrm{HC}(\mathrm{N}=27)$ & CSF & specific resonances in $\mathrm{AD}$ (unidentified) & [35] \\
\hline $\mathrm{AD}(\mathrm{N}=10), \mathrm{HC}(\mathrm{N}=34)$ & CSF & $\uparrow$ creatinine & [36] \\
\hline $\begin{array}{l}\operatorname{AD}(N=76), \operatorname{EAD}(N=26) \\
\operatorname{MCI}(N=33), H C(N=45)\end{array}$ & CSF & phenylalanine, glutamate, lactate, acetate, $\alpha$-hydroxyisovalerate, $\alpha$-hydroxybutyrate & [37] \\
\hline APP $\times$ PS1 $(N=6)$, WT $(N=6)$ & whole brain, plasma & $\begin{array}{l}\text { brain: } \downarrow N \text {-acetyl-aspartata, } \gamma \text {-aminobutyric acid, creatine, ascorbic acid } \\
\text { plasma: } \downarrow \text { glutamate, glutamine, methionine, acetate, citrate }\end{array}$ & [38] \\
\hline $\mathrm{A} \beta(\mathrm{N}=10), \mathrm{WT}(\mathrm{N}=10)$ & $\begin{array}{l}\text { whole blood, plasma } \\
\text { hippocampus }\end{array}$ & $\begin{array}{l}\text { whole blood: } \downarrow \text { niacinamide, hypoxanthine, citrate, lactate, pyruvate, creatine, choline, acetate, alanine, } \\
\text { phenylalanine, glycine, valine, tyrosine; } \uparrow \text { AMP } \\
\text { plasma: } \downarrow \text { citrate, pyruvate, creatine, phenylalanine, tyrosine, fatty acids, phospholipids glucose, formate, } \\
\text { glutamine } \\
\text { hippocampus: no significant differences }\end{array}$ & [39] \\
\hline $\begin{array}{l}\text { APP } \times \text { PS1 }(\mathrm{N}=27), \text { WT } \\
(\mathrm{N}=33)\end{array}$ & plasma, liver & $\begin{array}{l}\text { plasma: } \uparrow \text { lipoproteins, triglycerides } \\
\text { liver: } \uparrow \text { unsaturated fatty acids, } 3 \text {-hydroxybutyrate, aspartate, glutamine, uridine diphosphate, uridine } \\
\text { monophosphate, adenosine monophosphate; } \downarrow \text { alanine, lactate, betaine }\end{array}$ & [40] \\
\hline SAMP8 $(\mathrm{N}=5)$, SAMR1 $(\mathrm{N}=5)$ & serum & $\begin{array}{l}\downarrow \text { inosine, glucose, polyunsaturated fatty acids, choline, phosphocholine, HDL, LDL, 3-hydroxy-butyrate, } \\
\text { citrate, pyruvate; } \uparrow \text { lactate, saturated fatty acids, alanine, methionine, glutamine, VLDL }\end{array}$ & [41] \\
\hline $\begin{array}{l}\mathrm{APP}_{\operatorname{Tg} 2576}(\mathrm{~N}=3-5), \mathrm{WT} \\
(\mathrm{N}=3-5)\end{array}$ & urine & $\begin{array}{l}\uparrow 3 \text {-hydroxykynurenine, homogentisate, allantoin, acetate, trans-aconitate, tyrosine, hippurate, citrate, } \\
\text { urea; } \downarrow \text { trigonelline, } 2 \text {-oxoglutarate, dimethylamine, trimethylamine, threonine, } 1 \text {-methylnicotinamide }\end{array}$ & [42] \\
\hline \multicolumn{4}{|l|}{ Direct MS Analysis } \\
\hline $\mathrm{AD}(\mathrm{N}=22), \mathrm{HC}(\mathrm{N}=18)$ & serum & $\downarrow$ PUFA-PCs; $\uparrow$ SFA-PCs $\quad$ & {$[45]$} \\
\hline $\mathrm{AD}(\mathrm{N}=22), \mathrm{HC}(\mathrm{N}=18)$ & serum & $\begin{array}{l}\downarrow \text { PUFA-PLs, valine, glutamine, } N \text {-acetyl-glutamine, glutamate, histidine, arginine, dopamine, carnitine, } \\
\text { creatine; } \uparrow \text { SFA-PLs, free fatty acids, eicosanoids, triglycerides, choline, glycerophosphocholine, glucose }\end{array}$ & [46] \\
\hline $\mathrm{AD}(\mathrm{N}=22), \mathrm{HC}(\mathrm{N}=18)$ & serum & $\begin{array}{l}\downarrow \text { PUFA-PLs, oleamide, histidine, arginine, imidazole, taurine, guanidine, putrescine; } \uparrow \text { eicosanoids, } \\
\text { diglycerides, kynurenine }\end{array}$ & [47] \\
\hline $\mathrm{AD}(\mathrm{N}=19), \mathrm{HC}(\mathrm{N}=17)$ & serum & $\downarrow$ PUFA-PLs, LPLs, plasmalogens; $\uparrow$ SFA-PLs & [48] \\
\hline $\mathrm{AD}(\mathrm{N}=30), \mathrm{HC}(\mathrm{N}=30)$ & serum & $\begin{array}{l}\downarrow \text { fatty acid amides, urea, creatine, malate, taurine, dopamine, serotonin, } \uparrow \text { ceramides, diglycerides, free } \\
\text { fatty acids, alanine, picolinic acid }\end{array}$ & [49] \\
\hline $\begin{array}{l}\mathrm{AD}(\mathrm{N}=24), \mathrm{HC}(\mathrm{N}=6) \\
\mathrm{APP}_{\mathrm{V} 717 \mathrm{~F}}, \mathrm{APP}_{\mathrm{V} 717 \mathrm{~F}} \times \mathrm{APOE} \\
\mathrm{WT} \\
\mathrm{APP}_{\mathrm{sw}}, \mathrm{APP}_{\mathrm{sw}} \times \mathrm{APOE}, \mathrm{WT}\end{array}$ & $\begin{array}{l}\text { superior frontal cortex, } \\
\text { superior temporal cortex, } \\
\text { inferior parietal cortex, } \\
\text { cerebellum }\end{array}$ & $\downarrow$ plasmalogens & [50] \\
\hline $\mathrm{AD}(\mathrm{N}=17), \mathrm{HC}(\mathrm{N}=5)$ & $\begin{array}{l}\text { middle frontal gyrus, } \\
\text { superior temporal gyrus, } \\
\text { inferior parietal lobule, } \\
\text { hippocampus, subiculum, } \\
\text { entorhinal cortex }\end{array}$ & $\downarrow$ sulfatides; $\uparrow$ ceramides & [51] \\
\hline $\begin{array}{l}\mathrm{APP}_{\mathrm{V} 717 \mathrm{~F}}, \mathrm{APP}_{\mathrm{V} 717 \mathrm{~F}} \times \mathrm{APOE}, \mathrm{WT} \\
\mathrm{APP}_{\mathrm{sw}}, \mathrm{APP}_{\mathrm{sw}} \times \mathrm{APOE}, \mathrm{WT}\end{array}$ & cortex, cerebellum & $\downarrow$ sulfatides & [52] \\
\hline $\mathrm{AD}(\mathrm{N}=6), \mathrm{HC}(\mathrm{N}=8)$ & superior frontal gyrus & $\downarrow$ sulfatides & [53] \\
\hline $\mathrm{AD}(\mathrm{N}=26), \mathrm{HC}(\mathrm{N}=26)$ & plasma $>$ & $\downarrow$ long-chain sphingomyelins; $\uparrow$ ceramides & [54] \\
\hline $\begin{array}{l}\mathrm{AD}(\mathrm{N}=34), \mathrm{MCI}(\mathrm{N}=19), \mathrm{HC} \\
(\mathrm{N}=28)\end{array}$ & $\begin{array}{l}\text { CSF, frontal cortex grey } \\
\text { matter, frontal cortex } \\
\text { white matter }\end{array}$ & $\begin{array}{l}C S F: \downarrow \text { docosahexaenoic acid } \\
\text { grey matter: } \downarrow \text { plasmalogens, phosphatidylethanolamines, docosahexaenoic acid; } \uparrow \text { monoglycerides, } \\
\text { diglycerides, VLCFA } 26: 0 \\
\text { white matter: } \downarrow \text { phosphatidylethanolamines, sulfatides; } \uparrow \text { monoglycerides, diglycerides }\end{array}$ & [55] \\
\hline $\begin{array}{l}\operatorname{AD}(\mathrm{N}=90), \mathrm{MCI}(\mathrm{N}=77), \mathrm{HC} \\
(\mathrm{N}=51)\end{array}$ & serum & $\downarrow$ plasmalogens; $\uparrow$ diglycerides & [56] \\
\hline $\mathrm{AD}(\mathrm{N}=50), \mathrm{HC}(\mathrm{N}=50)$ & CSF & $\uparrow$ sphingomyelins, phosphatidylcholines & [57] \\
\hline $\begin{array}{l}\operatorname{AD}(\mathrm{N}=43), \mathrm{MCI}(\mathrm{N}=33), \mathrm{HC} \\
(\mathrm{N}=35)\end{array}$ & plasma & $\downarrow$ phosphatidylcholines; $\uparrow$ lyso-phosphatidylcholines & [58] \\
\hline
\end{tabular}


Table 1 (Continued)

\begin{tabular}{|c|c|c|c|}
\hline study population & sample & findings & Ref. \\
\hline $\begin{array}{l}\text { APP } \times \text { PS1 }(\mathrm{N}=30), \mathrm{WT} \\
(\mathrm{N}=30)\end{array}$ & serum & $\begin{array}{l}\downarrow \text { PUFA-PLs, LPLs, cholesteryl esters, free fatty acids, urea, serine, valine, threonine, pyroglutamate, } \\
\text { creatine, phosphoethanolamine, histidine, carnitine, glucose, tyrosine, tryptophan; } \uparrow \text { SFA-PLs, } \\
\text { eicosanoids, triglycerides, diglycerides, choline, GPE, inosine }\end{array}$ & [59] \\
\hline $\begin{array}{l}\text { APP } \times \text { PS } 1(N=10), \text { WT } \\
(N=10)\end{array}$ & urine & unidentified discriminant signals & [60] \\
\hline $\begin{array}{l}\text { APP } \times \text { PS } 1(N=30), \text { WT } \\
(\mathrm{N}=30)\end{array}$ & $\begin{array}{l}\text { hippocampus, cortex, } \\
\text { cerebellum, olfactory } \\
\text { bulb }\end{array}$ & $\begin{array}{l}\downarrow \text { PUFA-PLs, acyl-carnitines, steroids, adenine, UMP, AMP, urea, aspartate, dopamine, tyrosine, } \\
\text { homocarnosine; } \uparrow \text { LPLs, PL(18:0/20:4), 22:5PLs, 22:4-PLs, propionyl-carnitine, free fatty acids, } \\
\text { eicosanoids, uracil, hypoxanthine, xanthine, FAPy-adenine, adenosine, inosine, acetate, propionate, } \\
\text { pyruvate, alanine, choline, valine, G3P, phosphocholine, } N \text {-acetyl-spermidine, GPC, GPI }\end{array}$ & [61] \\
\hline $\begin{array}{l}\text { APP } \times \text { PS1 }(N=30), \text { WT } \\
(\mathrm{N}=30)\end{array}$ & $\begin{array}{l}\text { liver, kidney, spleen, } \\
\text { thymus }\end{array}$ & $\begin{array}{l}\text { alterations in the metabolism of phospholipids, fatty acids, acyl-carnitines, eicosanoids, acyl-glycerols, } \\
\text { steroids, amino acids, nucleotides, energy-related metabolites (changes depending on the tissue) }\end{array}$ & [62] \\
\hline $\begin{array}{l}\text { APP } \times \text { PS1 }(\mathrm{N}=8-9), \mathrm{WT} \\
(\mathrm{N}=8-9)\end{array}$ & brain, plasma & $\begin{array}{l}\text { longitudinal perturbations (6-18 months) in metabolism of phospholipids, amino acids, polyamines and } \\
\text { acyl-carnitines }\end{array}$ & [63] \\
\hline $\begin{array}{l}\text { APP } \times \text { PS } 1 \times \operatorname{IL} 4(N=7) \\
\text { APP } \times \text { PS } 1(N=7), \text { WT }(N=7)\end{array}$ & serum & $\begin{array}{l}\downarrow \text { urea, histamine, threonine, aspartate, urocanic acid, dopamine, citrulline, tyrosine; } \uparrow 1 \text {-methyl-histamine, } \\
\text { eicosanoids }\end{array}$ & [64] \\
\hline CRND8 $(\mathrm{N}=6), \mathrm{WT}(\mathrm{N}=6)$ & hippocampus & alterations in the homeostasis of arachidonic acid, glucose metabolism and fatty acid $\beta$-oxidation & [65] \\
\hline CRND8 $(\mathrm{N}=6)$, WT $(\mathrm{N}=6)$ & cerebellum & $\uparrow$ eicosanoids, amino acids and derivatives, xanthosine, xanthine, urate; $\downarrow$ inosine, guanosine & [66] \\
\hline \multicolumn{4}{|c|}{ Gas Chromatography-Mass Spectrometry } \\
\hline $\operatorname{AD}(\mathrm{N}=23), \mathrm{HC}(\mathrm{N}=21)$ & serum & $\begin{array}{l}\downarrow \text { valine, urea, aspartate, pyroglutamate, glutamine, phenylalanine, asparagine, ornithine, pipecolate, } \\
\text { histidine, tyrosine, palmitic acid, urate, tryptophan, stearic acid, cysteine; } \uparrow \text { lactate, } \alpha \text {-ketoglutarate, } \\
\text { isocitrate, glucose, oleic acid, adenosine, cholesterol }\end{array}$ & [67] \\
\hline $\begin{array}{l}\mathrm{AD}(\mathrm{N}=24), \mathrm{MCI}(\mathrm{N}=16), \mathrm{PD} \\
(\mathrm{N}=22), \mathrm{HC}(\mathrm{N}=8)\end{array}$ & exhaled breath & $\uparrow$ phenol (PD) & [68] \\
\hline $\mathrm{AD}(\mathrm{N}=9), \mathrm{HC}(\mathrm{N}=9)$ & $\begin{array}{l}\text { hippocampus, entorhinal } \\
\text { cortex, middle-temporal } \\
\text { gyrus, sensory cortex, } \\
\text { motor cortex, cingulate } \\
\text { gyrus, cerebellum }\end{array}$ & $\begin{array}{l}\text { changes in } 55 \text { metabolites, including energy related metabolites (glucose metabolism, TCA), amino acids, } \\
\text { nucleosides, and others }\end{array}$ & [69] \\
\hline $\begin{array}{l}\text { SAMP8 }(\mathrm{N}=5,2 \text { months; } \mathrm{N}=6 \text {, } \\
7 \text { months; } \mathrm{N}=7,12 \text { months })\end{array}$ & hippocampus & $\begin{array}{l}\uparrow \text { cholesterol, oleic acid, phosphoglyceride, } N \text {-acetyl-aspartate; } \downarrow \text { alanine, serine, glycine, aspartate, } \\
\text { glutamate, } \gamma \text {-aminobutyric acid, malic acid, butanedioic acid, fumaric acid, citric acid, pyroglutamic acid, } \\
\text { urea, lactic acid }\end{array}$ & [70] \\
\hline TASTPM $(\mathrm{N}=16)$, WT $(\mathrm{N}=5)$ & whole brain, plasma & $\begin{array}{l}\text { brain: } \uparrow \mathrm{D}-\text { fructose, valine, serine, threonine; } \uparrow \text { zymosterol } \\
\text { plasma: } \uparrow \mathrm{D} \text {-glucose, D-galactose, linoleica cid, arachidonic acid, palmitic acid, gluconic acid }\end{array}$ & [71] \\
\hline $\begin{array}{l}\operatorname{APP}_{\mathrm{Tg} 2576}(\mathrm{~N}=15), \mathrm{WT}(\mathrm{N}=17) \\
\mathrm{CRND} 8(\mathrm{~N}=9), \mathrm{WT}(\mathrm{N}=9) \\
\operatorname{APP}_{\mathrm{V} 717 \mathrm{I}}(\mathrm{N}=10), \mathrm{WT}(\mathrm{N}=12)\end{array}$ & urine & $\uparrow$ phenylacetone; $\downarrow$ 3-methylcyclopentanone, 4-methyl-6-hepten-3-one, 6-hydroxy-6-methyl-3-heptanone & [72] \\
\hline $\begin{array}{l}\text { CHO-A } \beta P_{695}(\mathrm{~N}=12,12 \mathrm{~h} \\
\mathrm{N}=10,24 \mathrm{~h}) \mathrm{CHO}-\mathrm{WT} \\
(\mathrm{N}=12,12 \mathrm{~h} ; \mathrm{N}=10,24 \mathrm{~h})\end{array}$ & culture media & $\begin{array}{l}\uparrow D \text {-glucose, malonate, methyl-malonate, citrate, } 2 \text {-oxoglutarate, serine, cystine; } \downarrow \text { succinate, pyruvate, } \\
\text { lysine, alanine, proline, ornithine, isoleucine glycine, tryptophan, threonine, leucine, uracil, glycerol }\end{array}$ & [73] \\
\hline \multicolumn{4}{|c|}{ Reversed-Phase Liquid Chromatography Mass Spectrometry (Hydrophobic metabolites) } \\
\hline $\mathrm{AD}(\mathrm{N}=10), \mathrm{HC}(\mathrm{N}=10)$ & plasma & $\downarrow$ demosterol & [74] \\
\hline $\begin{array}{l}\text { APOE2 }(\mathrm{N}=6), \text { APOE } 3(\mathrm{~N}=6) \\
\text { APOE4 }(\mathrm{N}=6)\end{array}$ & plasma, whole brain & alterations in phospholipids, sphingolipids, cholesterol and triglycerides & [75] \\
\hline $\begin{array}{l}\mathrm{APP} \times \mathrm{PS} 1 \\
\mathrm{APP} \times \mathrm{PS} 1 \times \mathrm{MAGL}^{-/-} \\
\mathrm{MAGL}^{-/-}, \text {WT }(\mathrm{N}=4-8)\end{array}$ & whole brain & $\begin{array}{l}A P P \times P S 1: \uparrow \text { monoglycerides, } N \text {-acylethanolamines, free fatty acids, eicosanoids, phosphatidic acids, } \\
\text { lysophosphatidylcholines, cholesteryl esters } \\
A P P \times P S 1 \times M A G L^{-/-}: \uparrow \text { arachidonoyl-glycerol; } \downarrow \text { arachidonic acid, prostaglandins, thromboxane } 2\end{array}$ & [76] \\
\hline $\begin{array}{l}\mathrm{AD}(\mathrm{N}=16), \mathrm{MCI}(\mathrm{N}=12), \mathrm{HC} \\
(\mathrm{N}=10)\end{array}$ & plasma & $\begin{array}{l}\uparrow 1 \text {-(9E-hexadecenoyl)-sn-glycero-3-phosphocholine, D-glucosaminide, glycocholic acid, } \\
\text { glycodeoxycholic acid, glycochenodeoxycholic acid }\end{array}$ & [77] \\
\hline $\mathrm{AD}(\mathrm{N}=20), \mathrm{HC}(\mathrm{N}=20)$ & plasma & $\downarrow$ lysophosphatidylcholines, tryptophan, phytosphingosine, dihydrosphingosine, hexadecasphinganine & [78] \\
\hline $\begin{array}{l}\mathrm{AD}(\mathrm{N}=36), \mathrm{MCI}(\mathrm{N}=48), \mathrm{HC} \\
(\mathrm{N}=40)\end{array}$ & plasma & $\downarrow$ long chain cholesteryl esters & [79] \\
\hline $\begin{array}{l}\mathrm{AD} / \mathrm{MCI}(\mathrm{N}=35), \text { Converters } \\
(\mathrm{N}=18), \mathrm{HC}(\mathrm{N}=53)\end{array}$ & plasma & $\begin{array}{l}\downarrow \text { phosphatidylcholines, lyso-phospholipids, serotonin, phenylalanine, proline, lysine, taurine, acyl- } \\
\text { carnitines, malate; } \uparrow \text { glycoursodeoxycholic acid, proline-asparagine }\end{array}$ & [80] \\
\hline $\begin{array}{l}\mathrm{AD}(\mathrm{N}=75), \mathrm{MCI}(\mathrm{N}=17), \mathrm{HC} \\
(\mathrm{N}=45)\end{array}$ & serum & $\begin{array}{l}\downarrow \text { PUFA-PLs, PUFA-SMs, sulfatides, monoglycerides, oleamide, histidine pregnenolone sulfate; } \uparrow \text { SFA- } \\
\text { PLs, SFA-SMs, ceramides, acyl-carnitines, phenyl-acetyl-glutamine }\end{array}$ & [81] \\
\hline $\operatorname{AD}(\mathrm{N}=660), \mathrm{MCI}(\mathrm{N}=583)$ & serum & $\begin{array}{l}\uparrow \text { sphinganine-1-phosphate, 7-ketocholesterol, 3-methoxytyrosine, deoxyribose-5-phosphate, } p \text { - } \\
\text { phenyllactic acid, lyso-phosphatidylcholine; } \downarrow \text { phenylalanine, ornithine, glutamate }\end{array}$ & [82] \\
\hline $\mathrm{AD}(\mathrm{N}=256), \mathrm{HC}(\mathrm{N}=218)$ & saliva & $\uparrow$ sphinganine-1-phosphate, ornithine, $p$-phenyllactic acid; $\downarrow$ inosine, 3 -dehydrocarnitine, hypoxanthine & [83] \\
\hline $\operatorname{AD}(\mathrm{N}=660), \mathrm{MCI}(\mathrm{N}=583)$ & saliva & $\begin{array}{l}\uparrow \text { cytidine, sphinganine-1-phosphate, ornithine, } p \text {-phenyllactic acid, pyroglutamate, glutamate, tryptophan; } \\
\downarrow \text { inosine, 3-dehydrocarnitine, hypoxanthine }\end{array}$ & [84] \\
\hline $\mathrm{AD}(\mathrm{N}=46), \mathrm{HC}(\mathrm{N}=37)$ & serum, urine & $\begin{array}{l}\text { serum: } \uparrow \text { palmitic amide, monoiodothyronine, antrimethyltridecanoic acid, PGE2 } \alpha \text { dimethyl amine, (6R)- } \\
\text { vitamin D3 6,19- (4-phenyl-1,2, 4-triazoline-3,5-dione); } \downarrow 1 \text {-methylinosine, } 16 \text {-bromo-9-hexadecenoic } \\
\text { acid, lysophosphatidylcholines, dihydrosphingosine, } N \text {-acetyl-glutamine, } 1 \alpha, 25 \text {-dihydroxy-2 } \alpha \text { - } \\
\text { (3-hydroxypropoxy) vitamin D3 } \\
\text { urine: } \uparrow N \text {-acryloylglycine, argininosuccinic acid, dethiobiotin, isobutyryl-L-carnitine, } \\
\text { 2-hydroxy- } N \text {-(2-hydroxyethyl)- } N, N \text {-dimethyl-1-dodecanaminium, L-aspartyl-4-phosphate, L-glutamine, } \\
\text { 5-L-glutamylglycine, azelaic acid, aminopterin, cytidine; } \downarrow \text { acetyl-carnitine, } \\
\text { estra-1,3,5(10),7-tetraene-3,17 } \alpha \text {-diol, } p \text {-cresol glucuronide, etiocholanolone glucuronide }\end{array}$ & [85] \\
\hline $\mathrm{AD}(\mathrm{N}=10), \mathrm{HC}(\mathrm{N}=10)$ & $\begin{array}{l}\text { frontal lobe, parietal lobe, } \\
\text { occipital lobe }\end{array}$ & $\uparrow$ spermidine, spermine, $N$-acetyl-spermidine, $N$-acetyl-spermine, putrescine & [86] \\
\hline
\end{tabular}


Table 1 (Continued)

\begin{tabular}{|c|c|c|c|}
\hline study population & sample & findings & Ref. \\
\hline $\begin{array}{l}\text { APP/tau }(\mathrm{N}=5,4 \text { months }) \text {, APP/ } \\
\text { tau }(\mathrm{N}=5,10 \text { months }), \text { APP/tau } \\
(\mathrm{N}=3,15 \text { months }) \text {, WT }(\mathrm{N}=5)\end{array}$ & whole brain, plasma & $\begin{array}{l}\text { brain: } \uparrow \text { cholesteryl esters, } 17,18 \text {-diHETE, } 19,20 \text {-diHDoPE; } \downarrow \text { PUFA-PLs, plasmalogens, sphingomyelins, } \\
\text { triglycerides, eicosanoids } \\
\text { plasma: } \uparrow \text { phospholipids, sphingomyelins, triglycerides, cholesteryl esters, eicosanoids }\end{array}$ & [87] \\
\hline $\begin{array}{l}\text { Sco }(N=10), \text { Sco }+ \text { HA } \\
(N=10), \operatorname{Sco}+\mathrm{LP}(\mathrm{N}=10) \\
\text { Sco }+ \text { HA }+ \text { LP }(N=10), W T \\
(N=10)\end{array}$ & whole brain & $\begin{array}{l}\uparrow \text { tyrosine, arginine, lyso-phosphatidylcholine- } 20: 5, \mathrm{PI}(16: 2) /(16: 1) /(16: 0), \text { S-adenosylhomocysteine, } \downarrow \downarrow \gamma \text { - } \\
\text { glutamylcysteine, } N \text {-acetylcysteine, DG(18:4)/(18:3)/(14:1), choline, taurine }\end{array}$ & {$[88]$} \\
\hline $\begin{array}{l}\text { A } \beta(N=6), A \beta+\text { Donezepil } \\
(N=6), A \beta+T G(N=6, \text { low- } \\
\text { medium-high doses }), \text { WT } \\
(N=10)\end{array}$ & plasma & $\begin{array}{l}\uparrow \text { proline, valine, tryptophan, lyso-phosphatidylcholines; } \downarrow \text { acyl-carnitines, phytosphingosine, } \mathrm{N} \text { - } \\
\text { eicosanoylethanolamine, fatty acids }\end{array}$ & [89] \\
\hline $\begin{array}{l}\text { APP } \times \text { PS } 1(N=10) \\
\text { APP } \times \text { PS } 1+\text { G-Rg1 }(\mathrm{N}=10) \\
\text { APP } \times \text { PS } 1+\text { G-Rg2 }(\mathrm{N}=10) \\
\text { WT }(\mathrm{N}=10)\end{array}$ & whole brain & $\begin{array}{l}\uparrow \text { hypoxanthine; } \downarrow \text { phytosphingosine, dihydrosphingosine, hexadecasphinganine, lyso- } \\
\text { phosphatidylcholines }\end{array}$ & {$[90]$} \\
\hline $\begin{array}{l}\mathrm{A} \beta(\mathrm{N}=10), \mathrm{A} \beta+\mathrm{G}-\mathrm{Rg} 1 \\
(\mathrm{~N}=10, \text { low-medium-high } \\
\text { doses }), \mathrm{A} \beta+\mathrm{G}-\mathrm{Rb} 1(\mathrm{~N}=10 \\
\text { low-medium-high doses }), \mathrm{WT} \\
(\mathrm{N}=10), \mathrm{WT}+\text { saline }(\mathrm{N}=10)\end{array}$ & plasma & $\uparrow$ phenylalanine; $\downarrow$ lyso-phosphatidylcholines, tryptophan, dihydrosphingosine & [91] \\
\hline $\begin{array}{l}\mathrm{D}-\mathrm{Gal} / \mathrm{Al}(\mathrm{N}=10), \mathrm{D}-\mathrm{Gal} / \\
\mathrm{Al}+\mathrm{KXS}(\mathrm{N}=10 \text { low- } \\
\text { medium-high doses), WT } \\
(\mathrm{N}=10)\end{array}$ & serum & $\begin{array}{l}\uparrow \text { lysine, allantoic acid, 5'-deoxyadenosine, N6-acetyl-L-lysine, GPC, 5-L-glutamyl-taurine, bile acids, } \\
\text { deoxyribose-5-phosphate, eicosanoids, hydrox-fatty acids, anserine, ubiquinone-1, QH2, arachidonic } \\
\text { acid, sulfatide; } \downarrow \text { uridine, leucine formylanthranilate, adenine, 4-imidazolone-5-propionic acid, } \\
\text { homovanillin, oxo-fatty acids, epimelibiose, } 3,4 \text {-dihydroxyphenylacetaldehyde, sphingosine, } \\
\text { phytosphingosine, adrenoyl ethanolamide, fatty acids }\end{array}$ & {$[92]$} \\
\hline \multicolumn{4}{|c|}{ Reversed-Phase Liquid Chromatography Mass Spectrometry (Hydrophilic metabolites) } \\
\hline $\mathrm{AD}(\mathrm{N}=17), \mathrm{HC}(\mathrm{N}=17)$ & CSF & 53 unidentified discriminant signals & [93] \\
\hline $\begin{array}{l}\text { AD I-II }(\mathrm{N}=7), \text { AD III-IV } \\
(\mathrm{N}=4), \text { AD V-VI }(\mathrm{N}=5), \mathrm{HC} \\
(\mathrm{N}=4)\end{array}$ & entorhinal cortex & $\downarrow$ dGMP, glycine, xanthosine, inosine diphosphate, deoxyguanosine; $\uparrow$ guanine & [94] \\
\hline $\mathrm{AD}(\mathrm{N}=15), \mathrm{HC}(\mathrm{N}=15)$ & CSF & $\begin{array}{l}\downarrow \text { norepinephrine, } \alpha \text {-tocopherols, } 3 \text {-methoxytyramine, ascorbate; } \uparrow 5 \text {-hydroxytryptophan, methoxy- } \\
\text { hydroxyphenyl glycol }\end{array}$ & [95] \\
\hline $\begin{array}{l}\mathrm{AD}(\mathrm{N}=40), \mathrm{MCI}(\mathrm{N}=36), \mathrm{HC} \\
(\mathrm{N}=38)\end{array}$ & CSF & $\uparrow$ methionine, 5-hydroxyindoleacetic acid, vanillylmandelic acid, xanthosine, glutathione, hypoxanthine & [96] \\
\hline $\begin{array}{l}\mathrm{AD}(\mathrm{N}=4 \text { young }), \mathrm{AD}(\mathrm{N}=4 \\
\text { old }), \mathrm{HC}(\mathrm{N}=3)\end{array}$ & frontal lobe & $\downarrow$ L-phenylalanine; $\uparrow$ L-lactate & [97] \\
\hline $\mathrm{MCI}(\mathrm{N}=20), \mathrm{HC}(\mathrm{N}=20)$ & saliva & $\begin{array}{l}\downarrow \text { taurine, peptides, 1,8-diazacyclotetradecane-2,9-dione, 4-(hydroxylamino)- } N, N \text {-dimethylaniline; } \\
\uparrow 2 \text {-amino-heptanoic acid/L-alanine- } n \text {-butyl ester/ } N \text {-methyl-isoleucine }\end{array}$ & [98] \\
\hline CRND8 $(\mathrm{N}=12), \mathrm{WT}(\mathrm{N}=12)$ & urine & $\begin{array}{l}\uparrow m e t h i o n i n e, \text { desaminotyrosine, phenylacetylglycine, dihydrouracil, ureidopropionic acid, thiocysteine; } \\
\downarrow \text { choline, taurine, N1-acetyl-spermidine, 5-hydroxy-indoleacetic acid, hydroxyphenylglycine }\end{array}$ & [99] \\
\hline \multicolumn{4}{|c|}{ Hydrophilic Interaction Liquid Chromatography Mass Spectrometry } \\
\hline $\mathrm{AD}(\mathrm{N}=15), \mathrm{HC}(\mathrm{N}=15)$ & neocortex & 76 unidentified discriminant signals & [100] \\
\hline $\mathrm{AD}(\mathrm{N}=20), \mathrm{HC}(\mathrm{N}=20)$ & plasma & 54 unidentified discriminant signals & [101] \\
\hline $\begin{array}{l}\text { MCI_AD }(\mathrm{N}=19), \text { MCI } \\
(\mathrm{N}=16), \mathrm{HC}(\mathrm{N}=37)\end{array}$ & plasma & $\begin{array}{l}\downarrow 4 \text {-amino-butanal, } \gamma \text {-aminobutyric acid, ornithine, } N \text {-acetyl-putrescine; } \uparrow \text { creatine, arginine, } \\
\text { methylthioadenosine, } N \text {-acetyl-spermidine, } N \text {-diacetyl-spermine, putrescine, spermidine, spermine }\end{array}$ & {$[102]$} \\
\hline $\begin{array}{l}\text { CRND8 }(\mathrm{N}=18 / 12,12 / 18 \\
\text { weeks), WT }(\mathrm{N}=12 / 12,12 / 18 \\
\text { weeks) }\end{array}$ & urine & $\begin{array}{l}55 \text { differential metabolites involved in the homeostasis of amino acids (e.g. tryptophan, tyrosine, } \\
\text { phenylalanine, lysine), fatty acids, purines and pyrimidines, ascorbate, and others }\end{array}$ & [103] \\
\hline \multicolumn{4}{|c|}{ Capillary Electrophoresis Mass Spectrometry } \\
\hline $\begin{array}{l}\mathrm{AD}(\mathrm{N}=23), \mathrm{MCI} \mathrm{AD}(\mathrm{N}=9) \\
\text { MCI_stable }(\mathrm{N}=22), \mathrm{SCI} \\
(\mathrm{N}=19)\end{array}$ & CSF & $\begin{array}{l}\uparrow \text { choline, valine, tripeptide, dimethylarginine, creatine, serine; } \downarrow \text { arginine, suberylglycine, carnitine, } \\
\text { histidine }\end{array}$ & [104] \\
\hline $\begin{array}{l}\mathrm{AD}(\mathrm{N}=42), \mathrm{MCI}(\mathrm{N}=14), \mathrm{HC} \\
(\mathrm{N}=37)\end{array}$ & serum & $\begin{array}{l}\uparrow \text { choline, creatinine, dimethylarginine, homocysteinyl-cysteine, acyl-carnitines, peptides; } \downarrow \text { creatine, } \\
\text { asparagine, methionine, histidine, carnitine, } N \text {-acetyl-spermidine, valeryl-carnitine }\end{array}$ & [105] \\
\hline $\begin{array}{l}\operatorname{AD}(\mathrm{N}=3), \text { FTLD }(\mathrm{N}=4), \text { LBD } \\
(\mathrm{N}=3), \mathrm{HC}(\mathrm{N}=9)\end{array}$ & serum, saliva & $\begin{array}{l}\text { serum: } \uparrow \beta \text {-alanine, creatinine, hydroxyproline, glutamine, isocitrate, cytidine } \\
\text { saliva: } \downarrow \text { arginine, tyrosine }\end{array}$ & [106] \\
\hline \multicolumn{4}{|c|}{ Reversed-Phase Liquid Chromatography/Gas Chromatography Mass Spectrometry } \\
\hline $\begin{array}{l}\operatorname{AD}(\mathrm{N}=47), \mathrm{MCI}(\mathrm{N}=143) \\
\mathrm{HC}(\mathrm{N}=46)\end{array}$ & serum & $\begin{array}{l}\text { baseline: } \downarrow \text { plasmalogens, phosphatidylcholines, sphingomyelins, sterols; } \uparrow \text { histamine } \\
\text { progression: } \uparrow 2,4 \text {-dihydroxybutanoic acid }\end{array}$ & [107] \\
\hline $\begin{array}{l}\mathrm{AD}(\mathrm{N}=57), \mathrm{MCI}(\mathrm{N}=58), \mathrm{HC} \\
(\mathrm{N}=57)\end{array}$ & plasma & $\begin{array}{l}\downarrow \text { fatty acids, dimethylglycine, glutamate, uridine, glyceraldehyde, butanedioic acid, } 2 \text {-butenedioic acid, } \\
\text { 5-oxoproline, taurine, hypotaurine, malate; } \uparrow \text { glutamine, } 2 \text {-aminoadipic acid, proline, cysteine, cytidine, } \\
\text { thymine, hypoxanthine, carbohydrates, citrate }\end{array}$ & [108] \\
\hline $\mathrm{AD}(\mathrm{N}=79), \mathrm{HC}(\mathrm{N}=51)$ & CSF & $\begin{array}{l}\uparrow \text { cysteine, tyrosine, phenylalanine, methionine, serine, pyruvate, taurine, creatinine, } \\
\text { cortisol, dopamine; } \downarrow \text { uridine }\end{array}$ & [109] \\
\hline $\mathrm{AD}(\mathrm{N}=40), \mathrm{HC}(\mathrm{N}=38)$ & CSF & 2 unidentified discriminant signals & {$[110]$} \\
\hline $\begin{array}{l}\text { APP } \times \text { PS1 }(N=30), \text { WT } \\
(N=30)\end{array}$ & serum & $\begin{array}{l}\text { alterations in the metabolism of phospholipids, sphingolipids, fatty acids, cholesterol and bile acids, } \\
\text { energy-related metabolites and amino acids }\end{array}$ & [111] \\
\hline $\begin{array}{l}\text { APP } \times \text { PS } 1(\mathrm{~N}=30), \text { WT } \\
(\mathrm{N}=30)\end{array}$ & $\begin{array}{l}\text { hippocampus, cortex, } \\
\text { striatum, cerebellum, } \\
\text { olfactory bulb }\end{array}$ & $\begin{array}{l}\text { alterations in the metabolism of phospholipids, sphingolipids, energy-related metabolites, amino acids } \\
\text { and nucleotides }\end{array}$ & [112] \\
\hline
\end{tabular}


Table 1 (Continued)

\begin{tabular}{|c|c|c|c|}
\hline study population & sample & findings & Ref. \\
\hline $\begin{array}{l}\text { APP } \times \text { PS1 }(N=30), \text { WT } \\
(\mathrm{N}=30)\end{array}$ & liver, kidney & $\begin{array}{l}\text { alterations in the metabolism of phospholipids, sphingolipids, fatty acids, acyl-carnitines, cholesterol and } \\
\text { bile acids, energy-related metabolites and amino acids }\end{array}$ & [113] \\
\hline $\begin{array}{l}\text { APP } \times \text { PS } 1(\mathrm{~N}=30), \mathrm{WT} \\
(\mathrm{N}=30)\end{array}$ & spleen, thymus & $\begin{array}{l}\text { alterations in the metabolism of phospholipids, sphingolipids, fatty acids, acyl-carnitines, energy-related } \\
\text { metabolites, amino acids and purines }\end{array}$ & [114] \\
\hline $\begin{array}{l}\mathrm{APP}_{\mathrm{Tg} 2576}(\mathrm{~N}=3), \operatorname{PS} 1(\mathrm{~N}=3) \\
\operatorname{APP} \times \operatorname{PS} 1(\mathrm{~N}=6), \mathrm{WT}(\mathrm{N}=6)\end{array}$ & hippocampus & $\begin{array}{l}\text { alterations in nucleotide, TCA cycle, energy transfer, carbohydrate, neurotransmitter and amino acid } \\
\text { metabolic pathways }\end{array}$ & [115] \\
\hline \multicolumn{4}{|c|}{ Reversed-Phase/Hydrophilic Liquid Chromatography Mass Spectrometry } \\
\hline $\begin{array}{l}\text { AD }(\mathrm{N}=21), \text { MCI_AD }(\mathrm{N}=12) \text {, } \\
\text { MCI_stable }(\mathrm{N}=21), \mathrm{HC} \\
(\mathrm{N}=21)\end{array}$ & CSF & $\begin{array}{l}\text { changes in levels of uracil, xanthine, uridine, tyrosyl-serine, methylsalsolinol, nonanoylglycine, } \\
\text { dopamine-quinone, caproic acid,vanylglycol, histidine, pipecolic acid, hydroxyphophinyl-piruvate, } \\
\text { creatinine, taurine, C16-sphingosine-1-phosphate, tryptophan, 5'-methylthioadenosine }\end{array}$ & [116] \\
\hline $\begin{array}{l}\mathrm{AD}(\mathrm{N}=30), \mathrm{MCI}(\mathrm{N}=30), \mathrm{HC} \\
(\mathrm{N}=30)\end{array}$ & plasma & $\downarrow$ sphingomyelins & [117] \\
\hline $\begin{array}{l}\mathrm{AD}(\mathrm{N}=15), \mathrm{MCI}(\mathrm{N}=15), \mathrm{HC} \\
(\mathrm{N}=15)\end{array}$ & plasma, CSF & changes in more than 150 metabolites (amino acids, energy-related metabolites, lipids, neurotransmitters) & [118] \\
\hline $\mathrm{AD}(\mathrm{N}=21), \mathrm{HC}(\mathrm{N}=19)$ & frontal cortex & $\begin{array}{l}\text { alterations in the metabolism of phospholipids and six metabolic pathways of the central metabolism: } \\
\text { Alanine, Aspartate, and Glutamate Metabolism; Arginine and Proline Metabolism; Cysteine and } \\
\text { Methionine Metabolism; Glycine, Serine, and Threonine Metabolism; Purine Metabolism; Pantothenate } \\
\text { and CoA Biosynthesis }\end{array}$ & [119] \\
\hline
\end{tabular}

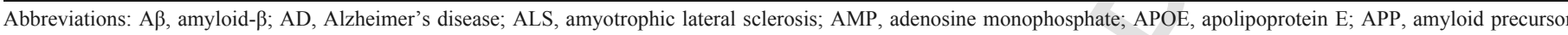

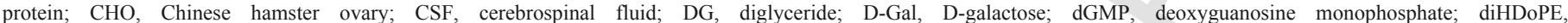

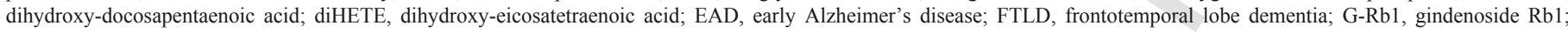

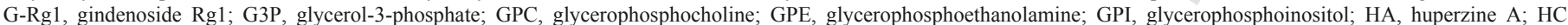

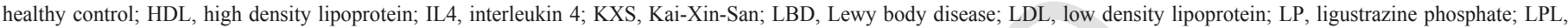

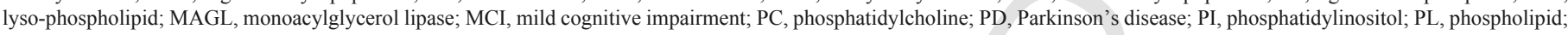

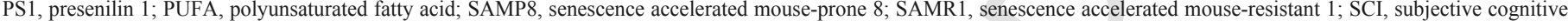

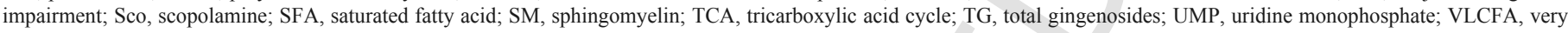
long chain fatty acid; VLDL, very low density lipoprotein; WT, wild type.

accurate classification models, thus evidencing the utility of non-invasive biological samples for studying pathological alterations underlying to this neurodegenerative disorder. Similarly, blood and hippocampus extracts from a mouse model produced by intracerebroventricular infusion of $A \beta$ peptides were examined in order to identify metabolic changes associated with the induction of neuroinflammatory processes [39]. Numerous perturbations were observed in blood levels of various metabolites involved in energy metabolism, but surprisingly no significant differences were detected in hippocampal metabolomic profiles. Furthermore, it is noteworthy that levels of these disrupted metabolites were normalized after treatment with gallic acid, a bioactive compound with anti-inflammatory properties, which supports that neuroinflammation is a primary trigger of $A \beta$-induced metabolomic alterations. In a more recent study, plasma and liver samples from APP $\times$ PS1 transgenic mice were subjected to NMR-based metabolomics in order to elucidate the possible involvement of peripheral organs in AD pathogenesis [40]. Significant changes were observed in levels of lipids, nucleotides and energy-related metabolites, thus demonstrating the occurrence of oxidative stress and impaired energy metabolism at systemic levels. Jiang et al. also found significant metabolic impairments in serum samples from the senescence accelerated mouse-prone 8 (SAMP8), which could be indicative of an abnormal homeostasis of glucose and lipids, as well as an attenuated protective function of inosine [41]. Finally, it should be noted that urine samples can also provide valuable information in $\mathrm{AD}$ research. In this context, Fukuhara et al. recorded ${ }^{1} \mathrm{H}$ NMR spectra of urine samples from $\mathrm{APP}_{\mathrm{Tg} 2576}$ transgenic mice aged between 4 and 15 months, which revealed significant metabolomic alterations indicative of oxidative stress in the onset of disease [42].

Accordingly, it could be concluded that NMR-based platforms exhibit a great potential for high-throughput metabolomics, which allows the simultaneous determination of various very important neurochemicals closely related to the pathogenesis of AD and other neurodegenerative disorders, including $\mathrm{N}$-acetyl-aspartate, myo-inositol, glutamate, $\gamma$-aminobutyric acid and taurine, among others. However, a major drawback of this metabolomic approach is its low sensitivity, which considerably limits the number of measurable metabolites, as shown in Table 1.

\subsubsection{Direct infusion/flow injection-mass spectrometry}

Similarly to NMR, metabolomics based on direct infusion or flow injection of crude extracts into a mass spectrometer can be used for high-throughput fingerprinting of samples in short times. However, it should be noted that MS is much more sensitive and selective than NMR, thus enabling the simultaneous determination of a wider range of metabolites in biological samples (Fig. 2). Thereby, since the publication of the first works describing the application of direct mass spectrometry analysis in metabolomics [43], numerous authors have demonstrated the potential of this technique as a suitable tool for fast and comprehensive metabolomic screening [44]. Due to the ion suppression caused by the direct introduction of whole samples into the ionization source, the most important findings in the majority of previously published DIMS-based studies in AD research are mainly associated with abnormalities in high-abundance lipids (Table 1). In this context, González-Domínguez et al. described the application of a metabolomic strategy based on a two-step sample treatment and DI-ESI-MS analysis for serum fingerprinting $[45,46]$. In order to obtain comprehensive metabolic profiles, serum samples were subjected to protein precipitation with organic solvents to get methanolic extracts containing polar metabolites, and then protein precipitates were again extracted to recover lipophilic compounds (Fig. 2). Notably, significant alterations were observed in circulating levels of different phospholipid (PL) classes, principally phosphatidylcholines (PC), with reduced content of species containing polyunsaturated fatty acids (PUFA) and increased levels of the corresponding saturated fatty acid (SFA)-derived lipids, which could be indicative of neural membrane breakdown processes. However, changes in other LMW metabolites were also detected, evidencing impairments in energy metabolism and neurotransmission, as well as the induction of oxidative stress in AD patients. Complementarily, serum samples from this cohort of patients were also examined using a lipidomic approach based on the extraction of lipids by means of a modification of the Bligh-Dyer 


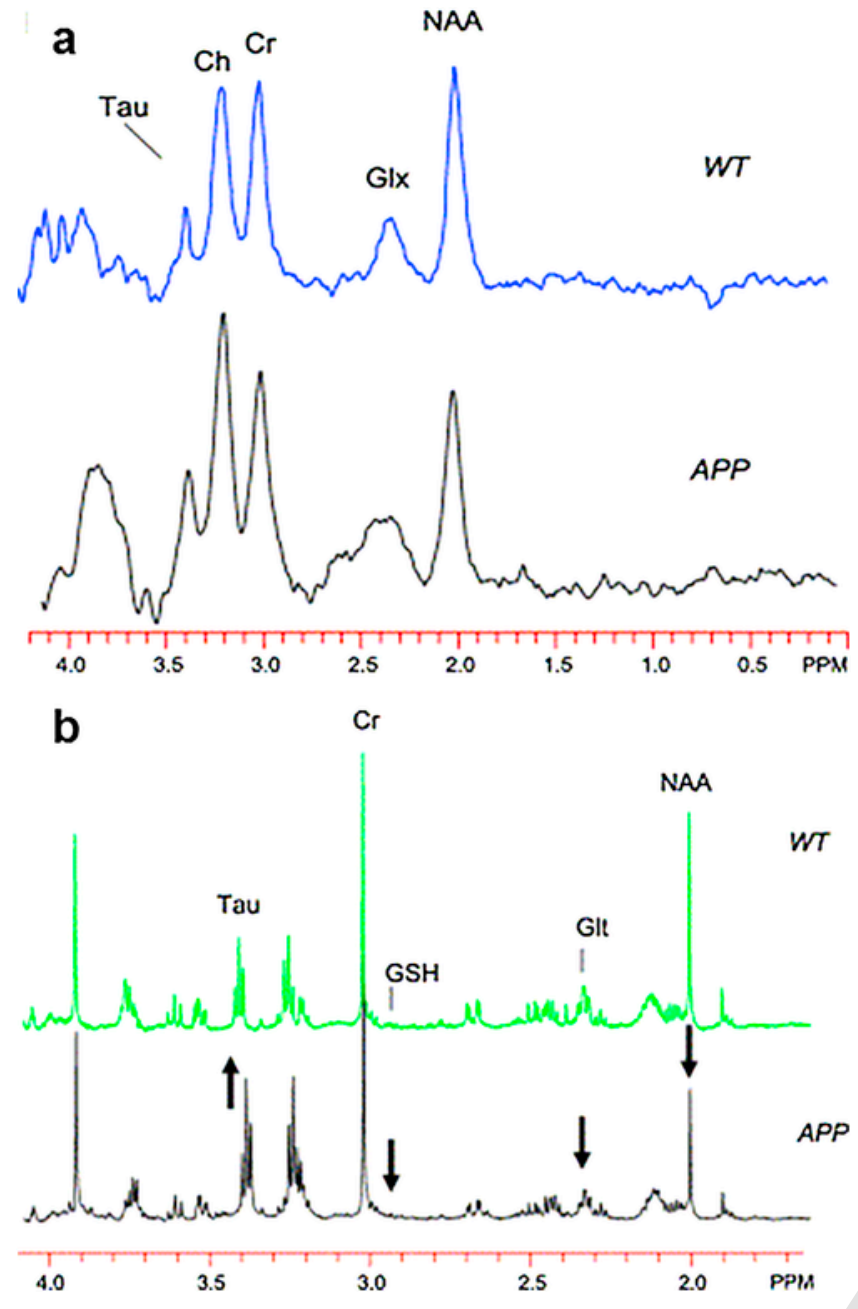

Fig. 1. Representative MRS (a) and NMR spectra (b) of cortex samples obtained from transgenic mice (APP) and wild type littermates (WT). Reprinted from Dedeoglu et al. [29] with permission from Elsevier.

method and subsequent analysis by DIMS [47]. Thereby, a large number of metabolites could be fingerprinted, including some neutral lipids not easily detectable by conventional metabolomic approaches, such as di- and triglycerides or cholesterol derivatives, among others. Besides a reduced content of PUFA-PLs, authors also observed a significant over-production of diglycerides, the final by-product of phospholipid hydrolysis via the action of phopholipases $\mathrm{C}$ and $\mathrm{D}$. Taking into account the huge evidence about the involvement of an abnormal metabolism of membrane lipids in $\mathrm{AD}$ pathogenesis, an analytical multi-platform was developed for performing a comprehensive characterization of the phospholipidome in order to get new insights about these disturbances [48]. For this, after DIMS-based metabolomics, serum phospholipids were profiled by RP-UHPLC coupled to elemental (inductively coupled plasma mass spectrometry) and molecular (quadrupole-time of flight) mass spectrometry. Thereby, it was demonstrated that impairments observed in phospholipid metabolism could have a multifactorial origin, involving different pathological processes such as the over-activation of phospholipases, imbalances in the levels of PUFA/SFA, oxidative stress or peroxysomal dysfunction, among others. In a more recent study, González-Domínguez et al. optimized a metabolomic platform based on flow injection in an APPI-MS system [49]. The APPI source provides complementary ionization capabilities to conventional ESI, thus allowing the simultaneous analysis of polar and non-polar compounds. The application of this analytical procedure to serum samples from $\mathrm{AD}$ patients revealed significant changes in levels of different lipid classes and other metabolites implicated in different neurotransmission systems. An interesting alternative to conventional DIMS for the comprehensive characterization of lipid alterations associated with $\mathrm{AD}$ is the multi-dimensional mass spectrometry-based shotgun lipidomic (MDMS-SL) platform. Numerous studies described the application of this strategy in plasma and brain samples, from both human patients and mouse models, thus evidencing significant perturbations in the homeostasis of plasmalogens [50], sulfatides [51-53], ceramides [51,54] and sphingomyelins [54]. More recently, Wood et al. used a novel lipidomic approach based on the extraction of lipids from CSF, brain and serum samples with a mixture of tert-butyl-methylether and methanol, and subsequent fingerprinting by ESI-Orbitrap-MS in order to investigate lipidomic alterations along the progression of AD from MCI $[55,56]$. In line with previous studies, significant changes were observed in levels of plasmalogens, phosphatidylethanolamines and diglycerides, among other compounds. Alternatively, other authors have proposed the use of targeted metabolomics based on the AbsoluteIDQ ${ }^{\mathrm{TM}}$ kit developed by Biocrates Life Sciences AG (Innsbruck, Austria), which allows the simultaneous quantification of endogenous metabolites from six different compound classes, including acyl-carnitines, amino acids, hexoses, phospholipids, sphingolipids and biogenic amines [57,58].

Direct MS analysis has also demonstrated a great utility for the investigation of AD-like pathology in different transgenic models. In this context, it should be noted the metabolomic multi-compartmental characterization of the APP $\times$ PS1 mouse model accomplished by González-Domínguez et al. in recent years. The combination of the previously described DI-ESI-MS and FI-APPI-MS methodologies enabled the identification of numerous disturbed pathways in serum samples from these mice, including perturbed phospholipid homeostasis, energy-related failures and oxidative stress, among others [59]. These findings are in agreement with earlier studies performed in cohorts of AD patients and healthy control subjects, thus confirming the potential of these transgenic mice to model this neurodegenerative disorder. In another work, the DIMS platform optimized for high-throughput serum fingerprinting was adapted for the analysis of urine samples because of the great interest of this biofluid in the clinical practice [60]. In order to minimize ion suppression caused by the high salt content of this matrix and to correct the inherent inter-individual variability, multiple sample preparation procedures and normalization strategies were assayed. Then, the optimized approach based on DIMS analysis of diluted urines and subsequent statistical data normalization was used to discriminate between APP $\times$ PS1 and WT mice. Various brain tissues were also examined by DIMS-based metabolomics in order to carry out an in situ investigation of neuropathological mechanisms related to $\mathrm{AD}$ pathogenesis and determine the regional specificity of these alterations [61]. Major differences were detected in cortex and hippocampus, but cerebellum and olfactory bulbs were also perturbed to a lesser extent. Finally, other peripheral organs were also analyzed in order to evaluate the systemic nature of this neurodegenerative disorder, including the liver, kidneys, spleen and thymus [62]. In this study, González-Domínguez et al. detected significant metabolic impairments associated with energy-production pathways, lipid homeostasis, oxidative stress or amino acid metabolism, in close similarity with findings previously described in brain, thus demonstrating the involvement of the peripheral system in AD. In this line, Pan et al. compared plasma and brain metabolomic profiles from APP $\times$ PS1 mice aged between 6 and 18 months using a targeted approach, and they found significant alter- 

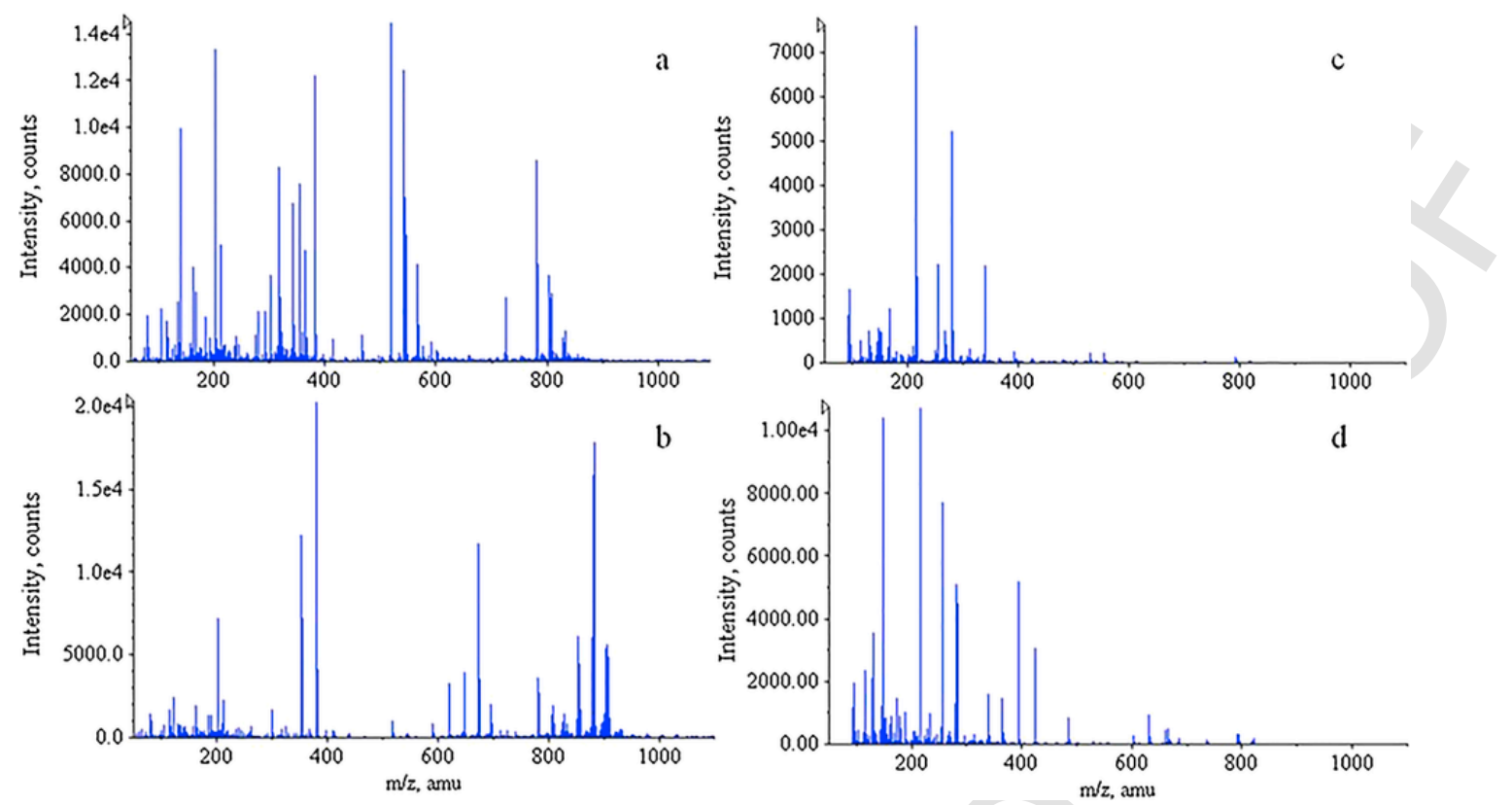

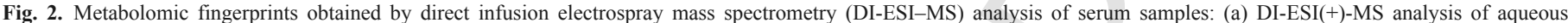

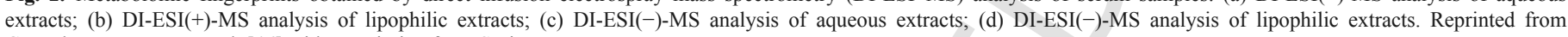
González-Domínguez et al. [46] with permission from Springer.

ations in phospholipid and acyl-carnitine homeostasis that follow a temporal sequence whereby changes in brain metabolites precede those in blood [63]. On the other hand, the triple transgenic mouse APP $\times$ PS $1 \times$ IL4-KO has been proposed as a suitable animal model to investigate the implication of inflammatory processes in the development of $\mathrm{AD}$. MS-fingerprinting of serum samples from these mice revealed significant changes in circulating levels of different eicosanoids, amino acids and related compounds, thus evidencing that depletion of interleukin 4 might potentiate AD pathology in the APP $\times$ PS1 model [64]. To conclude, Lin et al. employed ultra-high resolution mass spectrometry to identify metabolomic perturbations in hippocampus [65] and cerebellum [66] from CRND8 transgenic mice; and similarly to previous works with the APP $\times$ PS1 mouse model, metabolomic profiles exhibited characteristic features associated with abnormal homeostasis of arachidonic acid leading to the over-production of eicosanoids, as well as abnormal metabolism of amino acids, nucleotides and other metabolites.

\subsection{The volatile metabolome: gas chromatography-mass spectrometry}

Nowadays, the coupling GC-MS is still the most suitable alternative for the identification of volatile and semi-volatile compounds in complex biological samples, since it combines high separation efficiency with sensitive and reproducible mass spectral detection. This analytical platform has widely been employed for the profiling of several low molecular weight metabolite classes in AD research, such as free fatty acids, cholesterol derivatives or neurotransmitters, among others. However, the application of un-targeted GC-based metabolomics shows a greater potential to perform a more comprehensive characterization of the primary endogenous metabolome (i.e. amino acids, carbohydrates, organic acids, nucleotides) (Fig. 3). In this sense, González-Domínguez et al. identified 23 altered metabolites in serum samples from AD patients, some of which had not been previously described as potential biomarkers of this neurodegenerative disorder [67]. Pathway analysis revealed significant perturba- tions in the tricarboxylic acid cycle (TCA), several neurotransmitter systems, the urea cycle and many other pathways, thus confirming the utility of this metabolomic approach to monitor disturbances in central metabolic networks. Exhaled breath has also been proposed as an interesting biological matrix to investigate $\mathrm{AD}$ because blood changes can be partially transmitted to the alveolar breath through exchange in the lung. Volatile organic compounds were extracted by solid phase microextraction of breath samples from Alzheimer's disease, mild cognitive impairment and Parkinson's disease (PD) patients, and then were directly analyzed by GC-MS [68]. Thus, increased phenol content was observed in PD samples, but no significant differences were found in $\mathrm{AD}$ and MCI patients. More recently, widespread metabolic perturbations were detected in seven regions from human AD-brains, including those areas traditionally considered to be less affected by neuronal damage [69]. The most important findings were an abnormal metabolism of glucose, urea and various amino acids, which could occur prior to the brain volumetric loss and therefore could play a pivotal role in early pathogenesis of AD. Similarly, GC-MS hippocampal profiles from the SAMP8 mouse model also showed significant longitudinal alterations in levels of lipids, amino acids and energy-related metabolites along a 10 -months follow-up, from young mice ( 2 months old) to mature ( 7 months old) and finally aged mice (12 months old) [70]. In this line, $\mathrm{Hu}$ et al. detected a considerable number of disturbed metabolites in both brain and plasma samples from the TASTPM transgenic model, in agreement with previously described works [71]. The urinary volatile profile has also been employed to differentiate among three strains of APP transgenic mice [72]. For this, urine samples were fingerprinted by headspace GC-MS analysis, which enabled the identification of several odorants differentially expressed in these AD animal models. Finally, GC-based metabolomics was applied in a recent study focused on the investigation of the effects of A $\beta P P$ transgene on the Chinese hamster ovary (CHO) cellular line, as well as to confirm the therapeutic potential of two peroxisome proliferator-activated receptor gamma (PPAR $\gamma$ ) agonists [73]. Authors found that extracellular $A \beta$ accumulation is preceded by significant mitochondrial dysfunc- 

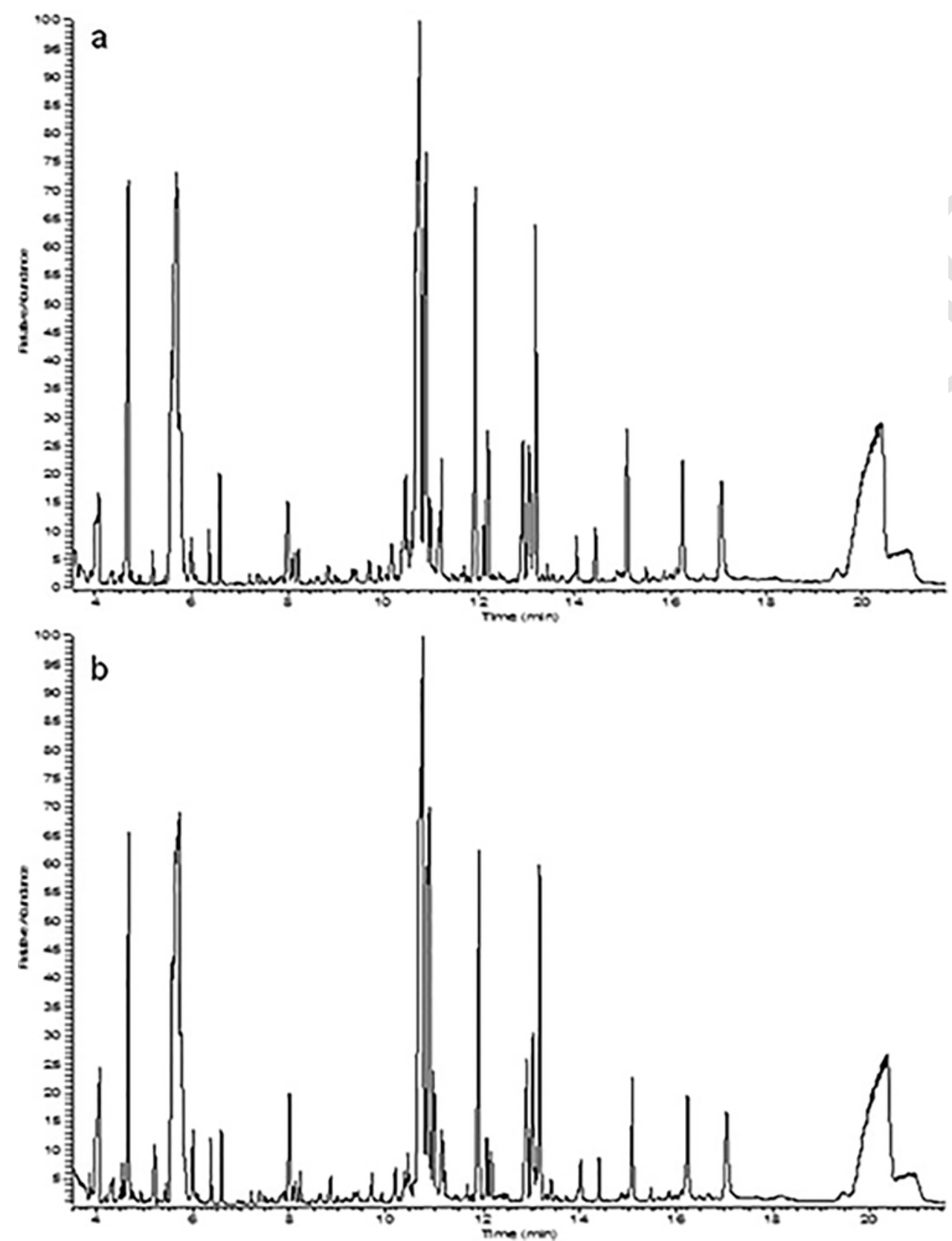

Fig. 3. Metabolomic profiles obtained by gas chromatography mass spectrometry (GC-MS) analysis of serum samples: (a) Alzheimer's disease; (b) healthy controls. Reprinted from González-Domínguez et al. [67] with permission from Elsevier.

tions, which can be attenuated after treating with these PPAR $\gamma$ agonists.

In summary, it is noteworthy that GC-MS exhibits a great potential to characterize the perturbations induced by $\mathrm{AD}$ in the volatile metabolome. Thus, despite the inherent drawbacks associated with this technique, such as the usual need of a derivatization step or the low sample throughput, a wide range of low molecular weight metabolites can be profiled with excellent sensitivity and reproducibility, including amino acids, organic acids, carbohydrates, fatty acids, nucleotides and some others (Fig. 3, Table 1).

\subsection{The non-polar metabolome: reversed phase liquid chromatography-mass spectrometry}

In the last years, liquid chromatography has replaced to gas chromatography as the most common separation technique in metabolomic applications because of its wide versatility for resolving various metabolite classes and higher sample treatment simplicity, without the need of a derivatization step. Particularly, reversed phase chromatography has become the first choice for the profiling of metabolites with medium and low polarity, which account up to $70 \%$ of the human metabolome according to the HMDB [15]. In this context, RP-HPLC-based lipidomics has extensively been proposed for the characterization of lipid abnormalities associated with the devel- 
opment of $\mathrm{AD}$ and other disorders. Sato et al. employed a metabolomic platform based of HPLC-APCI-MS for the unbiased determination of steroids in plasma samples from AD patients [74]. They found a considerably reduction in circulating demosterol levels, results that were additionally validated by subsequent targeted analysis. It has also been demonstrated that the apolipoprotein E (APOE) genotype has important repercussions on brain and plasma lipidomes of APOE $\varepsilon 2, \varepsilon 3$, and $\varepsilon 4$ knock-in mice, leading to significant alterations in the content of phospholipids, sphingolipids, cholesterol and triglycerides [75]. In another study, the combination of targeted and un-targeted lipidomic approaches revealed significant perturbations in the brain metabolism of endocannabinoids and eicosanoids in the APP $\times$ PS1 mouse model, which could be reversed by inactivating the monoacylglycerol lipase (MAGL) thus attenuating neuroinflammation and $A \beta$ production in these transgenic mice [76].

Alternatively, the use of ultra-high-performance liquid chromatography has largely emerged in the last decade because of its improved resolution, sensitivity and reduced analysis time (Fig. 4, Table 1). Plasma metabolomics has evidenced a close relationship between AD and the homeostasis of different lipid classes, including bile acids [77], lyso-phosphatidylcholines and sphingolipid precursors [78], long chain cholesteryl esters [79], and several phospholipids [80], among others. More recently, a comprehensive characterization of serum samples from $\mathrm{AD}$ and MCI patients was accomplished, revealing significant impairments in the metabolism of acyl-carnitines, lipids potentially implicated in the endocannabinoid system (i.e. oleamide and monoglycerides), as well as phospholipids and sphingolipids, wherein the chain length and degree of unsaturation of contained fatty acids plays a pivotal role [81]. Liang et al. also employed this analytical platform to differentiate $\mathrm{AD}, \mathrm{MCI}$ and healthy control subjects by analyzing serum [82] and saliva samples [83,84]. Re- ceiver operating characteristic (ROC) curves were performed in order to determine the diagnostic effectiveness of the identified discriminant metabolites, and the major contributors to these predictive models were sphinganine-1-phosphate and 7-ketocholesterol (serum, AD vs. MCI) [82], sphinganine-1-phosphate, ornithine and phenyl-lactic acid (saliva, AD vs. HC) [83], as well as sphinganine-1-phosphate and cytidine (saliva, AD vs. MCI) [84]. The comparative analysis of serum and urine samples also suggested significant lipid disorders mainly affecting the metabolism of lyso-phosphatidylcholines and sphingolipids, thus confirming the utility of these two non-invasive biofluids to discover AD biomarkers [85]. In another study, UHPLC-based metabolomics and targeted approaches were combined to investigate the involvement of an abnormal brain expression of the polyamine pathway in $\mathrm{AD}$ pathogenesis [86]. Tajima et al. also described the application of RP-UHPLC-MS to perform a global metabolomic analysis of brain and plasma samples from APP/tau transgenic mice, thus demonstrating a profound lipid dysregulation in this model [87]. To conclude, it is noteworthy that this metabolomic platform has also been proposed for studying the effects of some drugs on metabolic profiles of several AD animal models. The co-administration of huperzine A (HA) and ligustrazine phosphate (LP) showed a great potential to slow down the progression of AD pathology and regulate the levels of several potential biomarkers in brain samples from scopolamine (Sco) induced amnesic rats [88]. The intake of gingenosides, in form of individual species (G-Rg1, G-Rg2, G-Rb1) or as total gingenoside (TG) extracts, could also revert most of the metabolomic alterations observed in APP $\times$ PS1 and A $\beta$-lesioned mice [89-91]. Similarly, the treatment with different doses of the herbal Kai-Xin-San (KXS) significantly improved the cognitive impairment induced in rats by intraperitoneal injection of D-galactose (D-Gal) and intragastrical administration of aluminum chloride [92].

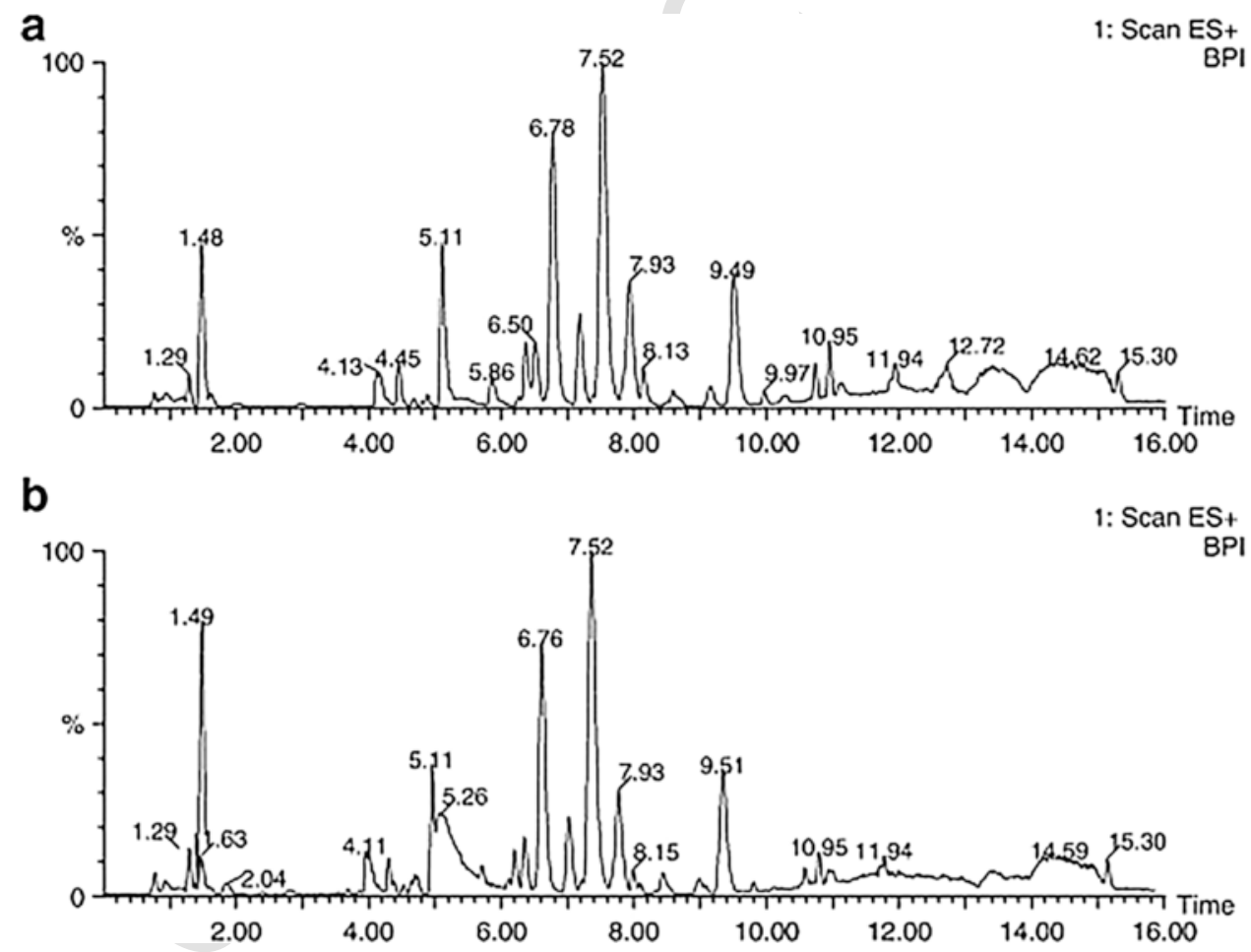

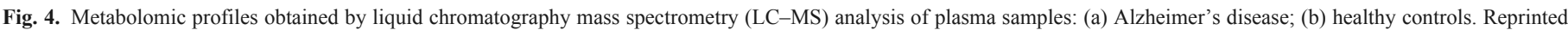
from $\mathrm{Li}$ et al. [78] with permission from Elsevier. 


\subsection{The hydrophilic metabolome: other strategies}

Hydrophilic metabolites are usually not retained in reversed phase LC columns, and only thermally stable compounds can be profiled by GC-MS after a time-consuming derivatization step. For this reason, complementary methodologies are necessary to expand the analytical coverage of MS-based techniques towards the polar metabolome. Different strategies have been proposed with the aim to improve the resolving power of RP-LC for hydrophilic compound analysis. In this sense, it should be noted that various manufacturers have developed novel RP columns with superior polar compound retention. Myint et al. optimized a metabolomic approach for the quantitative profiling of cationic metabolites in human CSF samples using a mixed-functional capillary column with RP and cation exchange resins [93]. Similarly, the combination of genomics, transcriptomics and UHPLC-based metabolomics in a Zorbax SB-Aq column enabled the elucidation of a deep deregulation of purine metabolism in entorhinal cortex of $\mathrm{AD}$ patients at different stages of disease [94]. Another alternative is the use of ion pairing reagents in order to increase the hydrophobicity, and consequently the retention, of polar metabolites. Kaddurah-Daouk et al. employed a metabolomic platform based on liquid chromatography with pentane sulfonic acid as ion-pairing agent and subsequent electrochemical array detection (ECA) to monitor redox-active metabolites in CSF from AD and MCI patients $[95,96]$. This technique enabled the detection of numerous metabolites involved in the homeostasis of tryptophan, tyrosine, phenylalanine, methionine and purine, thus revealing significant perturbations in pathways associated with neurotransmission, oxidative stress and one-carbon metabolism, among others. A third option is the derivatization of hydrophilic metabolites, which in addition to improve retention in RP columns can also help to increase sensitivity and isomer separation efficiency. In this context, chiral metabolomics based on the labeling with optically-active derivatization reagents and subsequent precursor ion scan chromatography of the derivatives has been proposed for the identification of enantiomeric amines and carboxyls as biomarker candidates for AD diagnosis [97]. Alternatively, dansylation of salivary and urinary metabolites has also been described for the investigation of metabolic abnormalities associated with AD pathogenesis $[98,99]$.

Hydrophilic interaction liquid chromatography (HILIC) is a relatively recent mode of separation that has gained a great popularity in the last years for the profiling of polar metabolites. This technique combines polar columns and mobile phases normally used in RP separations, with gradients starting at high organic concentrations. Under these conditions, hydrophilic compounds can be retained via partitioning onto a water-rich layer that is partially immobilized on the surface of the stationary phase, and then eluted by increasing the eluent water content. Thus, in contrast to conventional normal phase chromatography, HILIC shows a great compatibility with MS detection due to the high organic-content of mobile phases, which facilitates solvent evaporation and consequently improves ionization efficiency. Some preliminary studies demonstrated the potential of this analytical platform to differentiate $\mathrm{AD}$ patients and $\mathrm{HC}$ subjects by fingerprinting the polar metabolome of brain [100] and plasma samples [101]. However, despite a great number of ions were detected in both biological matrices, and statistical models built with these data were able to classify subjects with excellent accuracy, discriminant signals were not identified. More recently, HILIC-MS plasma metabolomics highlighted the central role of altered homeostasis of polyamines and arginine in the onset of $\mathrm{AD}$ and its progression from MCI [102], in line with previous studies [47,86]. In another work,
Tang et al. employed this metabolomic approach for analyzing hydrophilic metabolites in urine samples from CRND8 transgenic mice [103]. Significant changes were observed in urinary levels of multiple amino acids, fatty acids, nucleotides and other metabolites, thus evidencing impairments in the mitochondrial energy metabolism, tryptophan homeostasis and the induction of oxidative stress, among other pathological hallmarks.

Finally, the coupling CE-MS has also been described for the characterization of perturbations in the polar metabolome associated with AD pathogenesis in different biological fluids. Ibáñez et al. employed this separation technique to monitor metabolomic alterations related to $\mathrm{AD}$ progression in CSF from subjects with different cognitive status, including $\mathrm{AD}$ patients, $\mathrm{MCI}$ patients that progressed to $\mathrm{AD}$ within 2 years, MCI patients that remained stable after this follow-up period, and patients with subjective cognitive impairment (SCI) [104]. Thereby, a panel of LMW-hydrophilic metabolites was identified as potential biomarkers of disease progression, including choline, valine, a tripeptide, dimethylarginine, creatine, serine, arginine, suberylglycine, carnitine and histidine. Similarly, the analysis of serum samples revealed a close relationship between the development of AD and multiple pathological processes, such as oxidative stress, defects in energy metabolism, or vascular risk factors, among others [105]. Moreover, the combined CE-fingerprinting of serum and saliva samples enabled differentiating between different neurodegenerative dementias, including $\mathrm{AD}$, frontotemporal lobe dementia (FTLD) and Lewy body disease (LBD) [106].

\subsection{Comprehensive metabolomics: multi-platform approaches}

As a consequence of the selectivity of most of the previously described analytical techniques towards the profiling of specific metabolite classes, the use of metabolomic multi-platforms based on the combination of complementary tools is emerging in the last years as the most suitable option to achieve a comprehensive characterization of metabolite alterations underlying to complex phenotypes. With regards to $\mathrm{AD}$ research, two strategies have been previously described to this end, (i) the combination of reversed-phase liquid chromatography and gas chromatography; and (ii) the combination of orthogonal reversed-phase and hydrophilic interaction liquid chromatography.

\subsubsection{The combination of reversed-phase liquid chromatography and gas chromatography coupled to mass spectrometry}

The first multi-metabolomic investigation of AD pathogenesis was described by Orešič et al. in 2011 [107]. In this prospective study, serum samples from $\mathrm{AD}, \mathrm{MCI}$ and control subjects were subjected to lipidomic analysis by RP-UHPLC-MS and metabolomic analysis by $\mathrm{GC} \times \mathrm{GC}-\mathrm{MS}$. At the baseline, AD patients showed reduced circulating levels of plasmalogens, phosphatidylcholines, sphingomyelins and sterols; while 2,4-dihydroxybutanoic acid was identified as the most important predictor of dementia progression in the follow-up. More recently, two panels of potential diagnostic plasma markers were able to discriminate $\mathrm{AD}$ and $\mathrm{MCI}$ patients from normal controls, comprising metabolites involved in diverse biochemical pathways, such as energy metabolism, amino acid homeostasis or one-carbon metabolism, among others [108]. Complementarily, this metabolomic multi-platform has also been proposed for profiling cerebrospinal fluid samples. Czech et al. found numerous metabolite alterations that could be directly associated with perturbations in brain metabolism, principally in several neurotransmitter systems [109]. Then, ROC analysis was performed in order to evaluate the diagnostic performance of these discriminant metabolites. 
Thereby, it was observed that the combination of CSF levels of cortisol, uridine, cysteine and other amino acids provide predictive models with sensitivity and specificity above $80 \%$. In this line, another study compared the accuracy of metabolomics to discriminate AD patients from healthy controls with that provided by traditional pathological markers (i.e. A $\beta$ and tau) [110]. Two unidentified metabolic features showed promising capabilities as potential diagnostic markers, but further confirmatory and validation studies are required.

On the other hand, the combination of GC-MS and RP-UHPLC-MS has also been described for the comprehensive characterization of metabolic abnormalities induced in transgenic animal models of AD (Fig. 5). In order to complement and confirm preliminary findings obtained by DIMS analysis [59-62] (Section 4.1.2), González-Domínguez et al. employed this metabolomic multi-platform to investigate multiple biological compartments from the APP $\times$ PS1 mouse model. Numerous alterations were observed in the homeostasis of phospholipids, sphingolipids, fatty acids, cholesterol, acyl-carnitines, energy-related metabolites, amino acids, nucleotides and some other compounds affecting the whole organism, including serum [111], brain [112], metabolically active organs [113] and organs involved in the immune function [114], in line with previous findings. Similarly, Trushina et al. demonstrated that many of these impairments can also be detected in hippocampus from other transgenic animals [115].

\subsubsection{The combination of reversed-phase and hydrophilic} interaction liquid chromatography coupled to mass spectrometry

A second alternative to maximize metabolomic coverage is the combination of orthogonal RP and HILIC separation prior to the MS detection (Table 1). This strategy has been employed to analyze CSF samples from four diagnostic groups related to AD progression: healthy control subjects, stable MCI patients, MCI subjects who developed $\mathrm{AD}$ after a follow-up period of 2 years, and a group of patients with AD [116]. Significant alterations were observed in levels of 17 metabolites along the progression of disease among the four study groups, in line with previous findings obtained by CE-MS analysis [104]. In another study, Armirotti et al. optimized a metabolomic approach based on the extraction of plasma samples using a variation of the classical three-phase Bligh-Dyer procedure and subsequent analysis by RP/HILIC-UHPLC-MS [117]. The application of this metabolomic multi-platform to plasma samples from the $\mathrm{MCI} / \mathrm{AD}$ Italian prevention project evidenced a clear separation between MCI and control subjects, but surprisingly no differentiation was observed between AD patients and controls. The complementary analysis of plasma and CSF samples was also proposed with the aim to perform a more exhaustive investigation of pathological mechanisms underlying to $\mathrm{AD}$ [118]. Changes in more than 150 metabolites were detected, which could be related to 23 and 20 altered pathways in plasma and CSF, respectively. To conclude, the potential of combining orthogonal separation mechanisms has also been recently

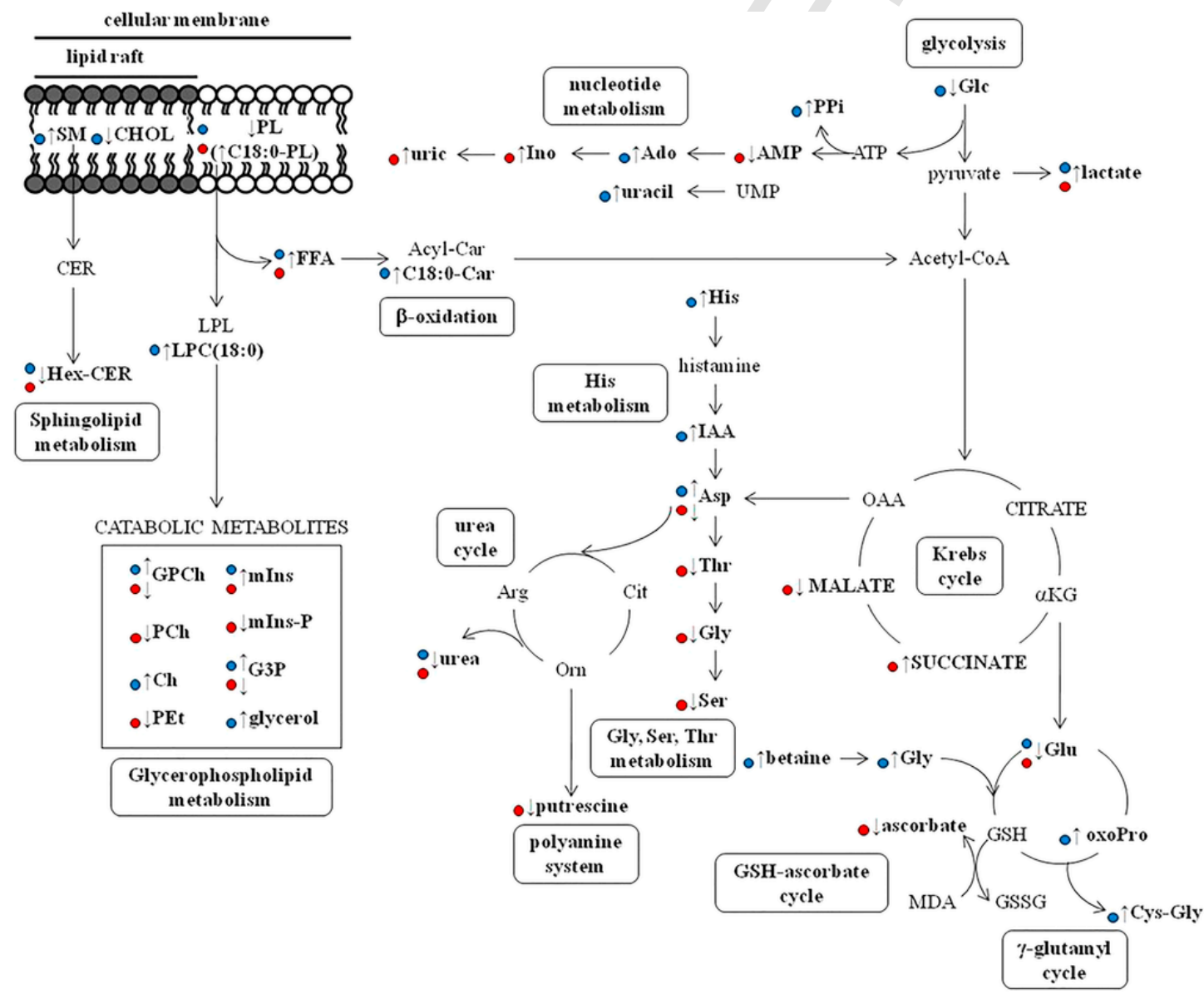

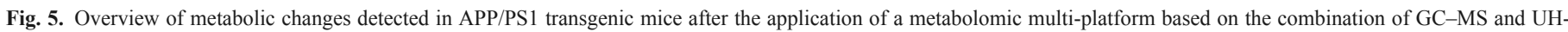
PLC-MS. Reprinted from González-Domínguez et al. [114] with permission from John Wiley and Sons. 
demonstrated by analyzing frontal cortex homogenates from AD and control subjects [119]. Authors found significant impairments in the homeostasis of phospholipids and six metabolic pathways of the central metabolism, and in conjunction with MS imaging confirmed a marked dysregulation of the mitochondrial aspartate metabolism.

\section{Critical discussion of existing metabolomic data: translating analytical results into the clinical practice}

The neuropathology of Alzheimer's disease has traditionally been associated with the formation of senile plaques and neurofibrillary tangles on brain, principally in cortical and hippocampal regions, leading to a gradual loss of neurons and synapses. However, there is great evidence supporting that this cognitive dysfunction may have a complex and heterogeneous origin. In this sense, it is noteworthy that impaired metabolism is increasingly being recognized as a pivotal player in the development of diseases usually not considered as "metabolic disorders", including cancer, neurodegenerative disorders and many others. Thereby, the application of metabolomic technologies has largely emerged in the last years with the aim to identify the metabolic signature of diseases in a comprehensive manner. Considering data presented in Table 1, it seems clear that multiple metabolic alterations could be involved in the pathogenesis and progression of Alzheimer's disease. Particularly, disturbed lipid metabolism has been proven to be a key hallmark of Alzheimer's disease, as reflected in abnormal levels of various lipid classes. Numerous metabolomic studies have previously reported significant changes in the content of phospholipids, sphingolipids and related catabolic metabolites in several biological samples, thus evidencing the occurrence of membrane destabilization processes. Similarly, cholesterol homeostasis also plays a pivotal role in $\mathrm{AD}$ pathogenesis, probably as a consequence of the implication of this compound on the formation of neuronal lipid rafts. Moreover, hyperlipidemia has been described as a very important risk factor for AD development, which is finally reflected in increased circulating levels of triglycerides and other neutral lipids. As would be expected, metabolomics-based investigations also revealed significant impairments in the biosynthesis of metabolites related to multiple neurotransmitter systems, such as the cholinergic, glutamatergic, GABAergic, serotonergic or dopaminergic systems. In addition, existing metabolomic data has also demonstrated profound impairments in numerous pathways related to energy metabolism, including glycolysis, the tricarboxylic acid cycle, $\beta$-oxidation of lipids, gluconeogenesis and ketogenesis, among others. This altered metabolic rate is usually accompanied by enhanced oxidative stress, which results in decreased levels of antioxidant compounds and increased markers of protein, lipid, and nucleic acid oxidation. Besides these most common metabolic alterations, there is also evidence of the involvement of other central pathways in $\mathrm{AD}$ pathogenesis, such as the urea cycle or the metabolism of purines, polyamines and bile acids among other metabolites, thus demonstrating the multifactorial character of this neurodegenerative disorder.

Although most of this previously published metabolomic research on Alzheimer's disease is focused on the elucidation of pathological mechanisms potentially related to disease etiology, some authors also investigated the utility of these discriminant metabolites as diagnostic biomarkers. In these studies, receiver operating characteristic (ROC) curve analysis is usually performed in order to validate the sensitivity and specificity of these candidate biomarkers. Czech et al. found that several combinations of three to five CSF metabolites could be used to discriminate $\mathrm{AD}$ patients from healthy control volunteers, but without any utility in the clinical practice since the statistical performance did not exceed to that provided by classical protein markers (i.e. A $\beta-42$ and tau) [109]. Conversely, in a more recent study it was demonstrated that GC-based metabolomic profiles provide similar discriminating potential to that showed by traditional pathological markers [110]. In this line, Laakso et al. reported that "the composition of CSF contains enough information of the neurological state of a given patient with a given dementia to be diagnosed with extremely high accuracy" [37]. Complementarily, the investigation of peripheral biological samples has also been proposed with the aim to identify simpler and cheaper biomarkers translatable to the clinical practice, including blood [58,74,108], saliva [83] and urine [85]. On the other hand, it is noteworthy that metabolomic data has also been employed to build predictive models able to monitor the progression of AD in the follow-up and to predict the conversion of mild cognitive impairment into Alzheimer's disease [80,82,84,107].

Anyway, none of these potential biomarkers identified through metabolomic approaches have reached the clinic because of various reasons. First, non-targeted metabolomics may produce conflicting results due to the lack of standardized protocols for sampling, analysis and data processing, as shown in Table 1. Furthermore, low population sizes usually employed in these studies reduce the statistical significance of findings obtained, so these preliminary data must be validated in large-scale human populations using targeted and quantitative analytical platforms before to be considered as clinical markers. Finally, it would be also interesting to extend these metabolomic investigations to other types of dementia with the aim to evaluate the specificity of these putative biomarkers against other neurodegenerative disorders.

\section{Conclusions \& perspectives}

Metabolomics has demonstrated a great potential to unravel the complex patho-biochemistry underlying to Alzheimer's disease, as summarized in the present review article. Various biological samples have been investigated, from both aged human cohorts and transgenic animal models, in order to get a deeper understanding of this disorder, including brain, cerebrospinal fluid, peripheral organs (e.g. liver, kidney, spleen, thymus), and several other biofluids such as plasma, serum, urine or saliva. Thereby, AD pathogenesis has been associated with widespread perturbations affecting multiple central metabolic pathways, including abnormal homeostasis of numerous lipid classes (e.g. phospholipids, sphingolipids, cholesterol, eicosanoids, acyl-carnitines), energy-related failures, disrupted neurotransmission, oxidative stress, and many others (Table 1). However, a major constraint of most of these previously published metabolomic studies is the lack of a reliable validation step of discriminant metabolites detected in the discovery phase using larger cohorts of patients. For this purpose, the inclusion of other types of dementias could be of great interest with the aim to evaluate the specificity of these candidate biomarkers, which has scarcely been considered to date $[27,68,106]$.

The application of a wide variety of metabolomic approaches has been described in AD research. Nuclear magnetic resonance can be employed for high-throughput profiling of some important endogenous metabolites, but its reduced sensitivity considerably hinders the analysis of complex samples. Alternatively, platforms based on direct mass spectrometry analysis (i.e. DI-MS, FI-MS) stand out as suitable tools for fast and non-discriminant metabolomic screening. On the other hand, the coupling of sensitive MS detection with orthogonal separation methods allows reducing the complexity of metabolomic profiles by focusing on specific metabolite classes. Reversed-phase liquid chromatography is nowadays the main workhorse in metabolomics because of its excellent performance for fingerprinting the non-polar metabolome. Conversely, gas chromatography-mass 
spectrometry provides high sensitivity and resolving power for the determination of primary low molecular weight metabolites (i.e. amino acids, organic acids, carbohydrates, amines). Moreover, other analytical techniques have also been proposed with the aim to expand the metabolomic coverage towards hydrophilic compounds without the recourse of a derivatization step, such as hydrophilic interaction liquid chromatography and capillary electrophoresis. As a consequence of the inherent analytical bias introduced by these techniques, it is noteworthy that none single platform is able to capture the entire metabolome. Thus, the combination of complementary approaches is emerging in recent years as the most suitable strategy to maximize the coverage of the AD-related metabolome. Basically, two different multi-platforms have been previously proposed in AD research: (i) RP-LC/GC-MS [107-115], and (ii) RP-LC/HILIC-MS [116-119]. However, metabolomic tools based on direct MS analysis have also gained a great popularity because of its high-throughput capability and wide metabolome coverage, which allows analyzing hundreds of samples per day in a comprehensive manner [44]. Thus, in the author's opinion, the combination of fast DIMS-based screening and subsequent fingerprinting via orthogonal hyphenated approaches could be the most suitable strategy with the aim to accomplish a global characterization of the human metabolome. DIMS analysis can be employed to carry out a preliminary investigation in order to visualize general trends and detect discriminant patterns among the study groups. Furthermore, its combination with ion mobility instruments could be an interesting alternative to allow the separation of isomers and increase the screening specificity through the measurement of collision cross section (CCS) values [120]. Then, low polarity compounds can be profiled by RP-UHPLC-MS. To date, most of the previously published UHPLC-MS-based studies have almost exclusively been performed via interfacing with electrospray ionization, but it should be noted that other ionization sources such as APCI and APPI can also provide complementary ionization capabilities to expand metabolomic coverage towards less polar compounds [47,59,74]. Furthermore, another important concern to be solved in order to increase the performance of this metabolomic platform is the improvement of existing spectral libraries and databases in order to facilitate the annotation of discriminant compounds [16]. Finally, several analytical techniques can be applied to monitor alterations in the hydrophilic metabolome. The couplings of HILIC and CE with mass spectrometry are relatively recent introduced tools in the metabolomic area for direct fingerprinting of polar and ionic compounds. However, despite the need of a previous derivatization step, GC-MS is still the most powerful choice for analyzing the LMW-metabolome due to its high sensitivity and separation efficiency, especially when multidimensional GC $\times$ GC is employed, as well as simpler peak identification through spectral libraries.

\section{References}

[1] C. Reitz, C. Brayne, R. Mayeux, Epidemiology of alzheimer disease, Nat. Rev. Neurol. 7 (2011) 137-152.

[2] R.C. Petersen, Mild cognitive impairment as a diagnostic entity, J. Intern. Med. 256 (2004) 183-194.

[3] K. Blennow, M.J. de Leon, H. Zetterberg, Alzheimer's disease, Lancet 368 (2006) 387-403.

[4] M.A. Smith, C.A. Rottkamp, A. Nunomura, A.K. Raina, G. Perry, Oxidative stress in Alzheimer's disease, Biochim. Biophys. Acta 1502 (2000) 139-144.

[5] E.E. Tuppo, H.R. Arias, The role of inflammation in Alzheimer's disease, Int. J. Biochem. Cell Biol. 37 (2005) 289-305.

[6] R. González-Domínguez, T. García-Barrera, J.L. Gómez-Ariza, Characterization of metal profiles in serum during the progression of Alzheimer's disease, Metallomics 9 (2014) 292-300.

[7] J.K. Morris, R.A. Honea, E.D. Vidoni, R.H. Swerdlow, J.M. Burns, Is Alzheimer's disease a systemic disease?, Biochim. Biophys. Acta 1842 (2014) $1340-1349$.
[8] B. Dubois, H.H. Feldman, C. Jacova, S.T. Dekosky, P. Barberger-Gateau, J. Cummings, A. Delacourte, D. Galasko, S. Gauthier, G. Jicha, K. Meguro, J. O’Brien, F. Pasquier, P. Robert, M. Rossor, S. Salloway, Y. Stern, P.J. Visser, P. Scheltens, Research criteria for the diagnosis of Alzheimer's disease: revising the NINCDS-ADRDA criteria, Lancet Neurol. 6 (2007) 734-746.

[9] D.S. Knopman, S.T. Dekosky, J.L. Cummings, H. Chui, J. Corey-Bloom, N. Relkin, G.W. Small, B. Miller, J.C. Stevens, Practice parameter: diagnosis of dementia (an evidence-based review). Report of the quality standards subcommittee of the American academy of neurology, Neurology 56 (2001) 1143-1153.

[10] W.B. Dunn, D.I. Broadhurst, H.J. Atherton, R. Goodacre, J.L. Griffin, Systems level studies of mammalian metabolomes: the roles of mass spectrometry and nuclear magnetic resonance spectroscopy, Chem. Soc. Rev. 40 (2011) $387-426$.

[11] D.B. Kell, P. Mendes, Snapshots of systems-metabolic control analysis and biotechnology in the post-genomic era, in: A. Cornish-Bowden, M.L. Cárdenas (Eds.), Technological and Medical Implications of Metabolic Control Analysis, Kluwer Academic Publishers, Dordrecht, 1999, pp. 3-25.

[12] C.W.W. Beecher, The human metabolome, in: G.G. Harrigan, R. Goodacre (Eds.), Metabolic Profiling: Its Role in Biomarker Discovery and Gene Function Analysis, Springer, New York, 2003, pp. 311-319.

[13] E. Trushina, M.M. Mielke, Recent advances in the application of metabolomics to Alzheimer's disease, Biochim. Biophys. Acta 1842 (2014) 1232-1239.

[14] R. Goodacre, S. Vaidyanathan, W.B. Dunn, G.G. Harrigan, D.B. Kell, Metabolomics by numbers: acquiring and understanding global metabolite data, Trends Biotechnol. 22 (2004) 245-252.

[15] D.S. Wishart, T. Jewison, A.C. Guo, M. Wilson, C. Knox, Y. Liu, Y. Djoumbou, R. Mandal, F. Aziat, E. Dong, S. Bouatra, I. Sinelnikov, D. Arndt, J. Xia, P. Liu, F. Yallou, T. Bjorndahl, R. Perez-Pineiro, R. Eisner, F. Allen, V. Neveu, R. Greiner, A. Scalbert, HMDB 3.0 - the human metabolome database in 2013, Nucleic Acids Res. 41 (2013) D801-D807.

[16] M. Vinaixa, E.L. Schymanski, S. Neumann, M. Navarro, R.M. Salek, O. Yanes, Mass spectral databases for LC/MS- and GC/MS-based metabolomics: state of the field and future prospects, Trends Anal. Chem. 78 (2016) 23-35.

[17] K. Bingol, R. Brüschweiler, Knowns and unknowns in metabolomics identified by multidimensional NMR and hybrid MS/NMR methods, Curr. Opin. Biotechnol. 43 (2016) 17-24.

[18] E. Rathahao-Paris, S. Alves, C. Junot, J.C. Tabet, High resolution mass spectrometry for structural identification of metabolites in metabolomics, Metabolomics 12 (2016) 10

[19] R. Craig-Schapiro, A.M. Fagan, D.M. Holtzman, Biomarkers of Alzheimer's disease, Neurobiol. Dis. 35 (2009) 128-140.

[20] K. Kantarci, S.D. Weigand, R.C. Petersen, B.F. Boeve, D.S. Knopman, J. Gunter, D. Reyes, M. Shiung, P.C. O'Brien, G.E. Smith, R.J. Ivnik, E.G. Tangalos, C.R. Jack Jr., Longitudinal ${ }^{1}$ H MRS changes in mild cognitive impairment and Alzheimer's disease, Neurobiol. Aging 28 (2007) 1330-1339.

[21] T. Watanabe, A. Shiino, I. Akiguchi, Hippocampal metabolites and memory performances in patients with amnestic mild cognitive impairment and Alzheimer's disease, Neurobiol. Learn. Mem. 97 (2012) 289-293.

[22] M. Marjanska, G.L. Curran, T.M. Wengenack, P.G. Henry, R.L. Bliss, J.F. Poduslo, C.R. Jack Jr., K. Ugurbil, M. Garwood, Monitoring disease progression in transgenic mouse models of Alzheimer's disease with proton magnetic resonance spectroscopy, Proc. Natl. Acad. Sci. U. S. A. 102 (2005) 11906-11910.

[23] J. Oberg, C. Spenger, F.H. Wang, A. Andersson, E. Westman, P. Skoglund, D. Sunnemark, U. Norinder, T. Klason, L.O. Wahlund, M. Lindberg, Age related changes in brain metabolites observed by ${ }^{1} \mathrm{H}$ MRS in APP/PS1 mice, Neurobiol. Aging 29 (2008) 1423-1433.

[24] W.E. Klunk, K. Panchalingam, J. Moossy, R.J. McClure, J.W. Pettegrew, $\mathrm{N}$-acetyl-L-aspartate and other amino acid metabolites in Alzheimer's disease brain: a preliminary proton nuclear magnetic resonance study, Neurology 42 (1992) 1578-1585.

[25] P. Mohanakrishnan, A.H. Fowler, J.P. Vonsattel, M.M. Husain, P.R. Jolles, P. Liem, R.A. Komoroski, An in vitro ${ }^{1} \mathrm{H}$ nuclear magnetic resonance study of the temporoparietal cortex of Alzheimer brains, Exp. Brain Res. 102 (1995) 503-510.

[26] P. Mohanakrishnan, A.H. Fowler, J.P. Vonsattel, P.R. Jolles, M.M. Husain, P Liem, L. Myers, R.A. Komoroski, Regional metabolic alterations in Alzheimer's disease: an in vitro ${ }^{1} \mathrm{H}$ NMR study of the hippocampus and cerebellum, J. Gerontol. A Biol. Sci. Med. Sci. 52 (1997) B111-B117.

[27] E.P. Botosoa, M. Zhu, C. Marbeuf-Gueye, M.N. Triba, F. Dutheil, C. Duyckäerts, P. Beaune, M.A. Loriot, L. Le Moye, NMR metabolomic of frontal cortex extracts: first study comparing two neurodegenerative diseases, Alzheimer disease and amyotrophic lateral sclerosis, IRBM 33 (2012) 281-286.

[28] S.F. Graham, C. Holscher, B.D. Green, Metabolic signatures of human Alzheimer's disease (AD): ${ }^{1} \mathrm{H}$ NMR analysis of the polar metabolome of post-mortem brain tissue, Metabolomics 10 (2014) 744-753.

[29] A. Dedeoglu, J.K. Choi, K. Cormier, N.W. Kowall, B.G. Jenkins, Magnetic resonance spectroscopic analysis of Alzheimer's disease mouse brain that ex- 
press mutant human APP shows altered neurochemical profile, Brain Res. 1012 (2004) 60-65.

[30] S. Balayssac, S. Déjean, J. Lalande, V. Gilard, M. Malet-Martino, A toolbox to explore NMR metabolomic data sets using the R environment, Chemometr. Intell. Lab. 126 (2013) 50-59.

[31] D.M. Forster, M.F. James, S.R. Williams, Effects of Alzheimer's disease transgenes on neurochemical expression in the mouse brain determined by ${ }^{1} \mathrm{H}$ MRS in vitro, NMR Biomed. 25 (2012) 52-58.

[32] R.M. Salek, J. Xia, A. Innes, B.C. Sweatman, R. Adalbert, S. Randle, E. McGowan, P.C. Emson, J.L. Griffin, A metabolomic study of the CRND8 transgenic mouse model of Alzheimer's disease, Neurochem. Int. 56 (2010) 937-943.

[33] D.C. Woo, S.H. Lee, D.W. Lee, S.Y. Kim, G.Y. Kim, H.S. Rhim, C.B. Choi, H.Y. Kim, C.U. Lee, B.Y. Choe, Regional metabolic alteration of Alzheimer's disease in mouse brain expressing mutant human APP-PS1 by ${ }^{1} \mathrm{H}$ HR-MAS, Behav. Brain Res. 211 (2010) 125-131.

[34] J. Lalande, H. Halley, S. Balayssac, V. Gilard, S. Déjean, R. Martino, B. Francés, J.M. Lassalle, M. Malet-Martino, ${ }^{1} \mathrm{H}$ NMR metabolomic signatures in five brain regions of the A $\beta$ PPswe Tg2576 mouse model of Alzheimer's disease at four ages, J. Alzheimers Dis. 39 (2014) 121-143.

[35] F. Kork, J. Holthues, R. Hellweg, V. Jankowski, M. Tepel, R. Öhring, I. Heuser, J. Bierbrauer, O. Peters, P. Schlattmann, W. Zidek, J. Jankowski, A possible new diagnostic biomarker in early diagnosis of Alzheimer's disease, Curr. Alzheimer Res. 6 (2009) 519-524.

[36] N.M. Jukarainen, S.P. Korhonen, M.P. Laakso, M.A. Korolainen, M. Niemitz, P.P. Soininen, K. Tuppurainen, J. Vepsäläinen, T. Pirttila, R. Laatikainen, Quantification of ${ }^{1} \mathrm{H}$ NMR spectra of human cerebrospinal fluid: a protocol based on constrained total-line-shape analysis, Metabolomics 4 (2008) $150-160$.

[37] M.P. Laakso, N.M. Jukarainen, J. Vepsäläinen, Diagnosis of dementias by high-field ${ }^{1} \mathrm{H}$ MRS of cerebrospinal fluid, J. Neurol. Neurosurg. Psychiatry 86 (2015) 1286-1290.

[38] S.F. Graham, C. Holscher, P. McClean, C.T. Elliott, B.D. Green, ${ }^{1}$ H NMR metabolomics investigation of an Alzheimer's disease (AD) mouse model pinpoints important biochemical disturbances in brain and plasma, Metabolomics 9 (2013) 974-983.

[39] E. Kim, Y.S. Jung, H. Kim, J.S. Kim, M. Park, J. Jeong, S.K. Lee, H.G. Yoon, G.S. Hwang, K. Namkoong, Metabolomic signatures in peripheral blood associated with Alzheimer's disease amyloid- $\beta$-induced neuroinflammation, J. Alzheimers Dis. 42 (2014) 421-433.

[40] J. Wu, B. Fu, H. Lei, H. Tang, Y. Wang, Gender differences of peripheral plasma and liver metabolic profiling in APP/PS1 transgenic AD mice, Neuroscience 332 (2016) 160-169.

[41] N. Jiang, X. Yan, W. Zhou, Q. Zhang, H. Chen, Y. Zhang, X. Zhang, NMR-based metabonomic investigations into the metabolic profile of the senescence-accelerated mouse, J. Proteome Res. 7 (2008) 3678-3686.

[42] K. Fukuhara, A. Ohno, Y. Ota, Y. Senoo, K. Maekawa, H. Okuda, M. Kurihara, A. Okuno, S. Niida, Y. Saito, O. Takikawa, NMR-based metabolomics of urine in a mouse model of Alzheimer's disease: identification of oxidative stress biomarkers, J. Clin. Biochem. Nutr. 52 (2013) 133-138.

[43] G. Madalinski, E. Godat, S. Alves, D. Lesage, E. Genin, P. Levi, J. Labarre, J.C. Tabet, E. Ezan, C. Junot, Direct introduction of biological samples into a LTQ-Orbitrap hybrid mass spectrometer as a tool for fast metabolome analysis, Anal. Chem. 80 (2008) 3291-3303.

[44] R. González-Domínguez, A. Sayago, M.A. Fernández-Recamales, Direct infusion mass spectrometry for metabolomic phenotyping of diseases, Bioanalysis 9 (2017) 131-148.

[45] R. González-Domínguez, T. García-Barrera, J.L. Gómez-Ariza, Metabolomic approach to Alzheimer's disease diagnosis based on mass spectrometry, Chem. Pap. 66 (2012) 829-835.

[46] R. González-Domínguez, T. García-Barrera, J.L. Gómez-Ariza, Using direct infusion mass spectrometry for serum metabolomics in Alzheimer's disease, Anal. Bioanal. Chem. 406 (2014) 7137-7148.

[47] R. González-Domínguez, T. García-Barrera, J.L. Gómez-Ariza, Metabolomic study of lipids in serum for biomarker discovery in Alzheimer's disease using direct infusion mass spectrometry, J. Pharm. Biomed. Anal. 98 (2014) 321-326.

[48] R. González-Domínguez, T. García-Barrera, J.L. Gómez-Ariza, Combination of metabolomic and phospholipid-profiling approaches for the study of Alzheimer's disease, J. Proteom. 104 (2014) 37-47.

[49] R. González-Domínguez, T. García-Barrera, J.L. Gómez-Ariza, Application of a novel metabolomic approach based on atmospheric pressure photoionization mass spectrometry using flow injection analysis for the study of Alzheimer's disease, Talanta 131 (2015) 480-489.

[50] X. Han, D.M. Holtzman, D.W. McKeel Jr., Plasmalogen deficiency in early Alzheimer's disease subjects and in animal models: molecular characterization using electrospray ionization mass spectrometry, J. Neurochem. 77 (2001) $1168-1180$
[51] X. Han, D.M. Holtzman, D.W. McKeel Jr., J.C. Morris, Substantial sulfatide deficiency and ceramide elevation in very early Alzheimer's disease: potential role in disease pathogenesis, J. Neurochem. 82 (2002) 809-818.

[52] H. Cheng, Y. Zhou, D.M. Holtzman, X. Han, Apolipoprotein E mediates sulfatide depletion in animal models of Alzheimer's disease, Neurobiol. Aging 31 (2010) 1188-1196.

[53] H. Cheng, M. Wang, J.L. Li, N.J. Cairns, X. Han, Specific changes of sulfatide levels in individuals with pre-clinical Alzheimer's disease: an early event in disease pathogenesis, J. Neurochem. 127 (2013) 733-738.

[54] X. Han, S. Rozen, S.H. Boyle, C. Hellegers, H. Cheng, J.R. Burke, K.A. Welsh-Bohmer, P.M. Doraiswamy, R. Kaddurah-Daouk, Metabolomics in early Alzheimer's disease: identification of altered plasma sphingolipidome using shotgun lipidomics, PLoS One 6 (2011) e21643.

[55] P.L. Wood, B.L. Barnette, J.A. Kaye, J.F. Quinn, R.L. Woltjer, Non-targeted lipidomics of CSF and frontal cortex grey and white matter in control, mild cognitive impairment, and Alzheimer's disease subjects, Acta Neuropsychiatry 27 (2015) 270-278.

[56] P.L. Wood, V.A. Locke, P. Herling, A. Passaro, G.B. Vigna, S. Volpato, G. Valacchi, C. Cervellati, G. Zuliani, Targeted lipidomics distinguishes patient subgroups in mild cognitive impairment (MCI) and late onset Alzheimer's disease (LOAD), BBA Clin. 5 (2016) 25-28.

[57] T. Koal, K. Klavins, D. Seppi, G. Kemmler, C. Humpel, Sphingomyelin $\mathrm{SM}(\mathrm{d} 18: 1 / 18: 0)$ is significantly enhanced in cerebrospinal fluid samples dichotomized by pathological amyloid- $\beta 42$, tau, and phospho-tau- 181 levels, $\mathrm{J}$ Alzheimers Dis. 44 (2015) 1193-1201

[58] K. Klavins, T. Koal, G. Dallmann, J. Marksteiner, G. Kemmler, C. Humpel, The ratio of phosphatidylcholines to lysophosphatidylcholines in plasma differentiates healthy controls from patients with Alzheimer's disease and mild cognitive impairment, Alzheimers Dement. 1 (2015) 295-302.

[59] R. González-Domínguez, T. García-Barrera, J. Vitorica, J.L. Gómez-Ariza, Application of metabolomics based on direct mass spectrometry analysis for the elucidation of altered metabolic pathways in serum from the APP/PS transgenic model of Alzheimer's disease, J. Pharm. Biomed. Anal. 107 (2015) 378-385.

[60] R. González-Domínguez, R. Castilla-Quintero, T. García-Barrera, J.L. Gómez-Ariza, Development of a metabolomic approach based on urine samples and direct infusion mass spectrometry, Anal. Biochem. 465 (2014) 20-27.

[61] R. González-Domínguez, T. García-Barrera, J. Vitorica, J.L. Gómez-Ariza, Metabolomic screening of regional brain alterations in the APP/PS1 transgenic model of Alzheimer's disease by direct infusion mass spectrometry, J. Pharm. Biomed. Anal. 102 (2015) 425-435.

[62] R. González-Domínguez, T. García-Barrera, J. Vitorica, J.L. Gómez-Ariza High throughput multi-organ metabolomics in the APP/PS1 mouse model of Alzheimer's disease, Electrophoresis 36 (2015) 2237-2249.

[63] X. Pan, M.B. Nasaruddin, C.T. Elliott, B. McGuinness, A.P. Passmore, P.G. Kehoe, C. Hölscher, P.L. McClean, S.F. Graham, B.D. Green, Alzheimer's disease-like pathology has transient effects on the brain and blood metabolome, Neurobiol. Aging 38 (2016) 151-163.

[64] R. González-Domínguez, T. García-Barrera, J. Vitorica, J.L. Gómez-Ariza Metabolomic research on the role of interleukin-4 in Alzheimer's disease, Metabolomics 11 (2015) 1175-1183.

[65] S. Lin, H. Liu, B. Kanawati, L. Liu, J. Dong, M. Li, J. Huang, P. Schmitt-Kopplin, Z. Cai, Hippocampal metabolomics using ultrahigh-resolution mass spectrometry reveals neuroinflammation from Alzheimer's disease in CRND8 mice, Anal. Bioanal. Chem. 405 (2013) 5105-5117.

[66] S. Lin, B. Kanawati, L. Liu, M. Witting, M. Li, J. Huang, P. Schmitt-Kopplin, Z. Cai, Ultrahigh resolution mass spectrometry-based metabolic characterization reveals cerebellum as a disturbed region in two animal models, Talanta 118 (2014) 45-53.

[67] R. González-Domínguez, T. García-Barrera, J.L. Gómez-Ariza, Metabolite profiling for the identification of altered metabolic pathways in Alzheimer's disease, J. Pharm. Biomed. Anal. 107 (2015) 75-81.

[68] P.W. Ko, K. Kang, J.B. Yu, J.S. Huh, H.W. Lee, J.O. Lim, Breath gas analysis for a potential diagnostic method of neurodegenerative diseases, Sens. Lett. 12 (2014) 1198-1202.

[69] J. Xu, P. Begley, S.J. Church, S. Patassini, K.A. Hollywood, M. Jüllig, M.A. Curtis, H.J. Waldvogel, R.L. Faull, R.D. Unwin, G.J. Cooper, Graded perturbations of metabolism in multiple regions of human brain in Alzheimer's disease: snapshot of a pervasive metabolic disorder, Biochim. Biophys. Acta 1862 (2016) 1084-1092.

[70] H. Wang, K. Lian, B. Han, Y. Wang, S.H. Kuo, Y. Geng, J. Qiang, M. Sun, M Wang, Age-related alterations in the metabolic profile in the hippocampus of the senescence-accelerated mouse prone 8: a spontaneous Alzheimer's disease mouse model, J. Alzheimers Dis. 39 (2014) 841-848.

[71] Z.P. Hu, E.R. Browne, T. Liu, T.E. Angel, P.C. Ho, E.C.Y. Chan, Metabonomic profiling of TASTPM transgenic alzheimer's disease mouse model, J. Proteome Res. 11 (2012) 5903-5913.

[72] B.A. Kimball, D.A. Wilson, D.W. Wesson, Alterations of the volatile metabolome in mouse models of Alzheimer's disease, Sci. Rep. 6 (2016) 19495 
[73] K.L. Chang, H.N. Pee, W.P. Tan, G.S. Dawe, E. Holmes, J.K. Nicholson, E.C Chan, P.C. Ho, Metabolic profiling of CHO-A $\beta$ PP695 cells revealed mitochondrial dysfunction prior to amyloid- $\beta$ pathology and potential therapeutic effects of both PPAR $\gamma$ and PPAR $\alpha$ agonisms for Alzheimer's disease, J. Alzheimers Dis. 44 (2015) 215-231.

[74] Y. Sato, I. Suzuki, T. Nakamura, F. Bernier, K. Aoshima, Y. Oda, Identification of a new plasma biomarker of Alzheimer's disease using metabolomics technology, J. Lipid Res. 53 (2012) 567-576.

[75] M.J. Sharman, G. Shui, A.Z. Fernandis, W.L.F. Lim, T. Berger, E. Hone, K. Taddei, I.J. Martins, J. Ghiso, J.D. Buxbaum, S. Gandy, M.R. Wenk, R.N. Martins, Profiling brain and plasma lipids in human APOE $\varepsilon 2, \varepsilon 3$, and $\varepsilon 4$ knock-in mice using electrospray ionization mass spectrometry, J. Alzheimers Dis. 20 (2010) 105-111.

[76] J.R. Piro, D.I. Benjamin, J.M. Duerr, Y.Q. Pi, C. Gonzales, K.M. Wood, J.W. Schwartz, D.K. Nomura, T.A. Samad, A dysregulated endocannabinoid-eicosanoid network supports pathogenesis in a mouse model of Alzheimer's disease, Cell Rep. 1 (2012) 617-623.

[77] N. Greenberg, A. Grassano, M. Thambisetty, S. Lovestone, C. Legido-Quigley, A proposed metabolic strategy for monitoring disease progression in Alzheimer's disease, Electrophoresis 30 (2009) 1235-1239.

[78] N.J. Li, W.T. Liu, W. Li, S.Q. Li, X.H. Chen, K.S. Bi, P. He, Plasma metabolic profiling of Alzheimer's disease by liquid chromatography/mass spectrometry, Clin. Biochem. 43 (2010) 992-997.

[79] P. Proitsi, M. Kim, L. Whiley, M. Pritchard, R. Leung, H. Soininen, I. Kloszewska, P. Mecocci, M. Tsolaki, B. Vellas, P. Sham, S. Lovestone, J.F. Powell, R.J. Dobson, C. Legido-Quigley, Plasma lipidomics analysis finds long chain cholesteryl esters to be associated with Alzheimer's disease, Transl. Psychiatry 5 (2015) e494.

[80] M. Mapstone, A.K. Cheema, M.S. Fiandaca, X. Zhong, T.R. Mhyre, L.H. MacArthur, W.J. Hall, S.G. Fisher, D.R. Peterson, J.M. Haley, M.D. Nazar, S.A. Rich, D.J. Berlau, C.B. Peltz, M.T. Tan, C.H. Kawas, H.J. Federoff, Plasma phospholipids identify antecedent memory impairment in older adults, Nat. Med. 20 (2014) 415-418.

[81] R. González-Domínguez, F.J. Rupérez, T. García-Barrera, C. Barbas, J.L. Gómez-Ariza, Metabolomic-driven elucidation of pathological mechanisms associated with Alzheimer's disease and mild cognitive impairment, Curr. Alzheimer Res. 13 (2016) 641-653.

[82] Q. Liang, H. Liu, T. Zhang, Y. Jiang, H. Xing, A.H. Zhang, Discovery of serum metabolites for diagnosis of progression of mild cognitive impairment to Alzheimer's disease using an optimized metabolomics method, RSC Adv. 6 (2016) 3586-3591.

[83] Q. Liang, H. Liu, T. Zhang, Y. Jiang, H. Xing, A.H. Zhang, Metabolomics-based screening of salivary biomarkers for early diagnosis of Alzheimer's disease, RSC Adv. 5 (2015) 96074-96079.

[84] Q. Liang, H. Liu, X. Lia, A.H. Zhang, High-throughput metabolomics analysis discovers salivary biomarkers for predicting mild cognitive impairment and Alzheimer's disease, RSC Adv. 6 (2016) 75499-75504.

[85] Y. Cui, X. Liu, M. Wang, L. Liu, X. Sun, L. Ma, W. Xie, C. Wang, S. Tang, D. Wang, Q. Wu, Lysophosphatidylcholine and amide as metabolites for detecting Alzheimer disease using ultrahigh-performance liquid chromatography-quadrupole time-of-flight mass spectrometry-based metabonomics, J. Neuropathol. Exp. Neurol. 73 (2014) 954-963.

[86] K. Inoue, H. Tsutsui, H. Akatsu, Y. Hashizume, N. Matsukawa, T. Yamamoto, T. Toyo'oka, Metabolic profiling of Alzheimer's disease brains, Sci. Rep. 3 (2013) 2364.

[87] Y. Tajima, M. Ishikawa, K. Maekawa, M. Murayama, Y. Senoo, T. Nishimaki-Mogami, H. Nakanishi, K. Ikeda, M. Arita, R. Taguchi, A. Okuno, R. Mikawa, S. Niida, O. Takikawa, Y. Saito, Lipidomic analysis of brain tissues and plasma in a mouse model expressing mutated human amyloid precursor protein/tau for Alzheimer's disease, Lipids Health Dis. 12 (2013) 68.

[88] J. Shi, Y. Wang, G. Luo, UPLC-TOF MS-based metabonomic study on coadministration of huperzine A and ligustrazine phosphate for treatment of Alzheimer's disease, Chromatographia 74 (2011) 827-832.

[89] Y. Gong, Y. Liu, L. Zhou, X. Di, W. Li, Q. Li, K. Bi, A UHPLC-TOF/MS method based metabonomic study of total ginsenosides effects on Alzheimer disease mouse model, J. Pharm. Biomed. Anal. 115 (2015) 174-182.

[90] N. Li, Y. Liu, W. Li, L. Zhou, Q. Li, X. Wang, P. He, UPLC/MS-based metabolomics investigation of the protective effectof ginsenosides $\mathrm{Rg} 1$ and Rg2 in mice with Alzheimer's disease, J. Ginseng Res. 40 (2016) 9-17.

[91] N. Li, L. Zhou, W. Li, Y. Liu, J. Wang, P. He, Protective effects of ginsenosides Rg1 and Rb1 on an Alzheimer's disease mouse model: a metabolomic study, J. Chromatogr. B 985 (2015) 54-61.

[92] H. Chu, A. Zhang, Y. Han, S. Lu, L. Kong, J. Han, Z. Liu, H. Sun, X. Wang, Metabolomics approach to explore the effects of Kai-Xin-San onAlzheimer's disease using UPLC/ESI-Q-TOF mass spectrometry, J. Chromatogr. B 1015-1016 (2016) 50-61.

[93] K.T. Myint, K. Aoshima, S. Tanaka, T. Nakamura, Y. Oda, Quantitative profiling of polar cationic metabolites in human cerebrospinal fluid by reversed-phase nanoliquid chromatography/mass spectrometry, Anal. Chem. 81 (2009) 1121-1129.
[94] B. Ansoleaga, M. Jové, A. Schlüter, P. Garcia-Esparcia, J. Moreno, A. Pujol, R. Pamplona, M. Portero-Otín, I. Ferrer, Deregulation of purine metabolism in Alzheimer's disease, Neurobiol. Aging 36 (2015) 68-80.

[95] R. Kaddurah-Daouk, S. Rozen, W. Matson, X. Han, C.M. Hulette, J.R. Burke, P.M. Doraiswamy, K.A. Welsh-Bohmer, Metabolomic changes in autopsy-confirmed Alzheimer's disease, Alzheimers Dement. 7 (2011) 309-317.

[96] R. Kaddurah-Daouk, H. Zhu, S. Sharma, M. Bogdanov, S.G. Rozen, W. Matson, N.O. Oki, A.A. Motsinger-Reif, E. Churchill, Z. Lei, D. Appleby, M.A. Kling, J.Q. Trojanowski, P.M. Doraiswamy, S.E. Arnold, Pharmacometabolomics Research Network, Alterations in metabolic pathways and networks in Alzheimer's disease, Transl. Psychiatry 3 (2013) e244.

[97] T. Takayama, T. Mochizuki, K. Todoroki, J.Z. Min, H. Mizuno, K. Inoue, H. Akatsu, I. Noge, T. Toyo'oka, A novel approach for LC-MS/MS-based chiral metabolomics fingerprinting and chiral metabolomics extraction using a pair of enantiomers of chiral derivatization reagents, Anal. Chim. Acta 898 (2015) 73-84.

[98] J. Zheng, R.A. Dixon, L. Li, Development of isotope labeling LC-MS for human salivary metabolomics and application to profiling metabolome changes associated with mild cognitive impairment, Anal. Chem. 84 (2012) 10802-10811.

[99] J. Peng, K. Guo, J. Xia, J. Zhou, J. Yang, D. Westaway, D.S. Wishart, L. Li, Development of isotope labeling liquid chromatography mass spectrometry for mouse urine metabolomics: quantitative metabolomic study of transgenic mice related to Alzheimer's disease, J. Proteome Res. 13 (2014) 4457-4469.

[100] S.F. Graham, O.P. Chevallier, D. Roberts, C. Holscher, C.T. Elliot, B.D. Green, Investigation of the human brain metabolome to identify potential markers for early diagnosis and therapeutic targets of Alzheimer's disease, Anal. Chem. 85 (2013) 1803-1811.

[101] K. Inoue, H. Tsuchiya, T. Takayama, H. Akatsu, Y. Hashizume, T. Yamamoto, N. Matsukawa, T. Toyo'oka, Blood-based diagnosis of Alzheimer's disease using fingerprinting metabolomics based on hydrophilic interaction liquid chromatography with mass spectrometry and multivariate statistical analysis, J. Chromatogr. B Anal. Technol. Biomed. Life Sci. 974 (2015) 24-34.

[102] S.F. Graham, O.P. Chevallier, C.T. Elliot, C. Hölscher, J. Johnston, B. McGuinness, P.G. Kehoe, A.P. Passmore, B.D. Green, Untargeted metabolomic analysis of human plasma indicates differentially affected polyamine and L-arginine metabolism in mild cognitive impairment subjects converting to Alzheimer's disease, PLoS One 10 (2015) e0119452.

[103] Z. Tang, L. Liu, Y. Li, J. Dong, M. Li, J. Huang, S. Lin, Z. Cai, Urinary metabolomics reveals alterations of aromatic amino acid metabolism of Alzheimer's disease in the transgenic CRND8 mice, Curr. Alzheimer Res. 13 (2016) 764-776.

[104] C. Ibánez, C. Simó, P.J. Martín-Álvarez, M. Kivipelto, B. Winblad, A. Cedazo-Mínguez, A. Cifuentes, Toward a predictive model of Alzheimer's disease progression using capillary electrophoresis-mass spectrometry metabolomics, Anal. Chem. 84 (2012) 8532-8540.

[105] R. González-Domínguez, A. García, T. García-Barrera, C. Barbas, J.L Gómez-Ariza, Metabolomic profiling of serum in the progression of Alzheimer's disease by capillary electrophoresis-mass spectrometry, Electrophoresis 35 (2014) 3321-3330.

[106] M. Tsuruoka, J. Hara, A. Hirayama, M. Sugimoto, T. Soga, W.R. Shankle, M. Tomita, Capillary electrophoresis-mass spectrometry-based metabolome analysis of serum and saliva from neurodegenerative dementia patients, Electrophoresis 34 (2013) 2865-2872.

[107] M. Orešič, T. Hyötyläinen, S.K. Herukka, M. Sysi-Aho, I. Mattila, T. Seppänan-Laakso, V. Julkunen, P.V. Gopalacharyulu, M. Hallikainen, J. Koikkalainen, M. Kivipelto, S. Helisalmi, J. Lötjönen, H. Soininen, Metabolome in progression to Alzheimer's disease, Transl. Psychiatry 1 (2011) e57.

[108] G. Wang, Y. Zhou, F.J. Huang, H.D. Tang, X.H. Xu, J.J. Liu, Y. Wang, Y.L. Deng, R.J. Ren, W. Xu, J.F. Ma, Y.N. Zhang, A.H. Zhao, S.D. Chen, W. Jia, Plasma metabolite profiles of Alzheimer's disease and mild cognitive impairment, J. Proteome Res. 13 (2014) 2649-2658.

[109] C. Czech, P. Berndt, K. Busch, O. Schmitz, J. Wiemer, V. Most, H. Hampel, J. Kastler, H. Senn, Metabolite profiling of Alzheimer's disease cerebrospinal fluid, PLoS One 7 (2012) e31501.

[110] A.A. Motsinger-Reif, H. Zhu, M.A. Kling, W. Matson, S. Sharma, O. Fiehn, D.M. Reif, D.H. Appleby, P.M. Doraiswamy, J.Q. Trojanowski, R. Kaddurah-Daouk, S.E. Arnold, Comparing metabolomic and pathologic biomarkers alone and in combination for discriminating Alzheimer's disease from normal cognitive aging, Acta Neuropathol. Commun. 1 (2013) 28.

[111] R. González-Domínguez, T. García-Barrera, J. Vitorica, J.L. Gómez-Ariza, Deciphering metabolic abnormalities associated with Alzheimer's disease in the APP/PS1 mouse model using integrated metabolomic approaches, Biochimie 110 (2015) 119-128.

[112] R. González-Domínguez, T. García-Barrera, J. Vitorica, J.L. Gómez-Ariza, Region-specific metabolic alterations in the brain of the APP/PS1 transgenic mice of Alzheimer's disease, Biochim. Biophys. Acta 1842 (2014) 2395-2402.

[113] R. González-Domínguez, T. García-Barrera, J. Vitorica, J.L. Gómez-Ariza, Metabolomic investigation of systemic manifestations associated with 
Alzheimer's disease in the APP/PS1 transgenic mouse model, Mol. BioSyst. 11 (2015) 2429-2440.

[114] R. González-Domínguez, T. García-Barrera, J. Vitorica, J.L. Gómez-Ariza, Metabolomics reveals significant impairments in the immune system of the APP/PS1 transgenic mice of Alzheimer's disease, Electrophoresis 36 (2015) 577-587.

[115] E. Trushina, E. Nemutlu, S. Zhang, T. Christensen, J. Camp, J. Mesa, A. Siddiqui, Y. Tamura, H. Sesaki, T.M. Wengenack, P.P. Dzeja, J.F. Poduslo, Defects in mitochondrial dynamics and metabolomic signatures of evolving energetic stress in mouse models of familial Alzheimer's disease, PLoS One 7 (2012) e32737.

[116] C. Ibañez, C. Simo, D.K. Barupal, O. Fiehn, M. Kivipelto, A.

Cedazo-Mínguez, A. Cifuentes, A new metabolomic workflow for early detection of Alzheimer's disease, J. Chromatogr. A 1302 (2013) 65-71.

[117] A. Armirotti, A. Basit, N. Realini, C. Caltagirone, P. Bossù, G. Spalletta, D. Piomelli, Sample preparation and orthogonal chromatography for broad polar- ity range plasma metabolomics: application to human subjects with neurodegenerative dementia, Anal. Biochem. 455 (2014) 48-54.

[118] E. Trushina, T. Dutta, X.M. Persson, M.M. Mielke, R.C. Petersen, Identification of altered metabolic pathways in plasma and CSF in mild cognitive impairment and Alzheimer's disease using metabolomics, PLoS One 8 (2013) e63644.

[119] G. Paglia, M. Stocchero, S. Cacciatore, S. Lai, P. Angel, M.T. Alam, M. Keller, M. Ralser, G. Astarita, Unbiased metabolomic investigation of Alzheimer's disease brain points to dysregulation of mitochondrial aspartate metabolism, J. Proteome Res. 15 (2016) 608-618.

[120] P. Dwivedi, P. Wu, S.J. Klopsch, G.J. Puzon, L. Xun, H.H. Hill Jr., Metabolic profiling by ion mobility mass spectrometry (IMMS), Metabolomics 4 (2008) $63-80$ 